\title{
X-ray measurements of growth rates at a gas interface accelerated by shock waves
}

\author{
R. Bonazzaa) and B. Sturtevant \\ Graduate Aeronautical Laboratories, California Institute of Technology, Pasadena, California 91125
}

(Received 8 December 1995; accepted 7 May 1996)

\begin{abstract}
A new experimental technique to measure the density of a high atomic number gas at a shock-accelerated interface has been developed and demonstrated. It is based on the absorption of $\mathrm{x}$ rays by the high atomic number gas, and it was implemented in a vertical square shock tube. The object of the study was the turbulent entrainment and mixing of shock-accelerated air/xenon interfaces prepared by retracting a metal plate, initially separating the two gases, prior to the release of the shock wave. Interfaces of two types, quasi-sinusoidal and nominally flat, were examined. The amplitude of large wavelength $(25-100 \mathrm{~mm})$ perturbations on the interface, and the thickness of the interface were measured. An integral definition for the interface mean line was adopted, making it possible to study and time evolution of the individual Fourier modes of the perturbations. A new integral definition for the interface thickness was proposed, making it feasible to study for the first time the time evolution of the thickness of quasi-sinusoidal interfaces. Images of interfaces after interacting with a series of weak waves reverberating between the interface and the shock tube end wall were obtained. The perturbations are studied at the late stages of their evolution, when their amplitude is no longer small compared to their wavelength. Consequently, the measured growth rates of the modal amplitudes are smaller than those predicted by the impulsive model based on the small amplitude approximation. In the case of nominally flat interfaces, the thickness is observed to grow linearly at rates comparable to values previously reported. (c) 1996 American Institute of Physics. [S1070-6631(96)00809-4]
\end{abstract}

\section{INTRODUCTION}

Instabilities can arise at the interface between fluids of different densities, under a variety of conditions. Examples are: the Rayleigh-Taylor instability, ${ }^{1,2}$ which amplifies the perturbations on an interface between fluids of different densities, accelerated in the direction from the lighter to the heavier; the Richtmyer-Meshkov instability, ${ }^{3,4}$ driven by the interaction of a shock wave with a similar interface, regardless of the direction of propagation of the shock; and the Landau-Darrieus instability, in which a mass flux across an interface between fluids of different densities destabilizes the interface. ${ }^{5}$ Common to these instabilities is the driving mechanism, viz. baroclinic generation of vorticity at the interface. The Kelvin-Helmholtz instability ${ }^{6}$ also arises between stratified fluids, as a consequence of relative tangential, rather than normal, motion between the layers.

The events that take place upon interaction of a shock wave with the interface between two gases of different physical properties can be grouped into two coupled categories: The effects generated on the wave by the interface, viz. the refraction and distortion of the shock wave, ${ }^{7-10}$ and the effects induced by the shock on the interface, viz. the setting in motion of the interface and the baroclinic generation of vorticity wherever the product $\nabla \rho \times \nabla p$ is nonzero. The vorticity deposited on the interface at the time of the interaction with the shock persists there after shock refraction, leading to

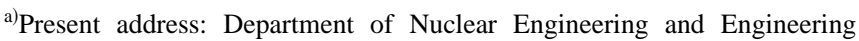
Physics, University of Wisconsin, Madison, Wisconsin 53706. Telephone: (608) 165-2337, fax: (608) 262-6707, electronic mail: bonazza@engr.wisc.edu
}

the distortion of the interface. At the early times, the evolution of the interface can be described by linearized equations of motion. Both an impulsive model ${ }^{3}$ and more recent and detailed linearized analytical treatments ${ }^{11}$ show that, during this linear phase, the growth rate asymptotically reaches a constant value proportional to the wave number. Since the baroclinic generation of vorticity is proportional to the magnitude of the density gradient, the perturbations of an interface of finite thickness grow more slowly than those on a discontinuous one. When the perturbation amplitude is no longer small compared to the wavelength, nonlinearities become important, causing the shape of the perturbations to distort from the original one and the growth rates to become smaller than those predicted for the linear stage. If very fine scale perturbations are present, the large wave numbers saturate almost immediately, generating small-scale turbulent motions whose macroscopic consequence is the entrainment of the fluids from both sides and the thickening of the interface. For nominally flat interfaces, exhibiting no largewavelength perturbations (compared to their thickness), this mixing process is actually the only measurable consequence of the original shock-interface interaction. If both large and small wavelength perturbations are present, both amplitude growth and interface thickening are observed. In the present experiment, an interface is prepared inside a vertical shock tube and a planar shock wave is incident on the interface from above. Perturbations are generated in part on the interface at the time it is constructed in the shock tube, and, more importantly, they are induced on the interface by the acoustic waves generated by the interaction of the reflected shock wave with the turbulent boundary layer behind the transmitted shock. 
Other experiments have been carried out in this laboratory. ${ }^{12-14}$ High-speed schlieren cinematography was used to examine the effects of density ratio, incident shock strength, and initial thickness on the evolution of the interface. The most important results were (i) the observation of a theoretically predicted reduction of the amplitude growth rate at interfaces of finite thickness; (ii) the measurement of a thickness growth rate of discontinuous interfaces almost an order of magnitude smaller than what had been previously reported, ${ }^{15}$ and (iii) the observation of wall vortices, generated by the interaction of the reflected shock with the boundary layers produced by the incident shock, whose presence partially obstructed the view of the interface.

The present work is carried out in a vertical, square shock tube. A new technique for quantitative visualization of the density field is developed, based on x-ray absorption by the heavy gas at the interface (xenon), in turn used to implement a quantitative densitometry procedure. The objective is to measure the distortion and thickness of the interface. Data are extracted from the x-ray negatives by digitization and by a calibration of the digital image. An integral definition of mean interface shape (first proposed by Pham ${ }^{16}$ ) is employed and an integral definition of interface thickness is introduced. The interface shape is decomposed into its Fourier components, and their time evolution is studied.

Schlieren visualization experiments are performed on air/xenon and air/ $\mathrm{SF}_{6}$ interfaces, to compare the $\mathrm{x}$-ray and schlieren imaging techniques. All experiments are performed using interfaces of initial finite thickness, with pseudo-single scale and multi-scale perturbations. The Mach number of the incident shock waves is either $M_{i}=1.32$ or $M_{i}=1.52$. The interface is studied upon interaction with the incident shock, the first reflected shock, and a series of re-reflected shock waves.

\section{PREVIOUS RELATED WORK}

\section{A. Richtmyer-Meshkov instability}

Richtmyer's ${ }^{3}$ impulsive model for the case of a discontinuous interface with single-wavelength perturbations accelerated by a single shock travelling from a fluid of density $\rho_{1}$ into one of density $\rho_{2}$ predicts an amplitude growth rate given by

$$
\frac{d \eta}{d t}=k[v] A \eta_{0},
$$

where $\eta$ is the amplitude and $k$ the wave number of the perturbations, $\eta_{0}$ the amplitude at time $t=0,[v]$ is the velocity jump induced at the interface, and $A=\left(\rho_{2}-\rho_{1}\right) /\left(\rho_{2}+\rho_{1}\right)$ is the Atwood number. Use of postshock values $A^{\prime}$ and $\eta_{0}^{\prime}$ leads to $5 \%$ agreement between the impulsive model and numerical simulations of the linearized equations of motion. Fraley ${ }^{17}$ used the Laplace transform to solve the same equations in wave number space. Yang et al. ${ }^{11}$ compared the impulsive model to their numerical solution to the linearized equations of motion over a larger parameter space than previously done by Richtmyer, identifying both regions of agreement and disagreement. Mikaelian ${ }^{18}$ compared his results from direct numerical simulations of the nonlinear equations to both the impulsive model and Fraley's solution. Holmes et al. ${ }^{19}$ applied a front-tracking numerical scheme to solve the nonlinear equations of motion and compared their findings to both the impulsive model and the results of the linear theory. Typically, both the impulsive model and the linear theory predict growth rates larger than those observed experimentally. ${ }^{20,21}$ The front-tracking numerical simulation produces growth rates in much closer agreement to the experimental values, but the results do not seem to reach an asymptotic steady state in contrast to what some of the experiments ${ }^{21}$ show.

Mikaelian ${ }^{22}$ studied the interface of finite thickness (in this work indicated with $\delta$ ) under shock acceleration using an $(N+1)$-layer model. Another approach (as e.g., in Ref. 13) is to modify Richtmyer's impulsive model with a growth reduction factor proposed by Duff et al. ${ }^{23}$ for the constant acceleration case:

$$
\frac{d \eta}{d t}=\frac{k[v] A^{\prime}}{\psi} \eta_{0}^{\prime} .
$$

In general, $\psi=\psi(A, \delta / \lambda)$; in the above expression the value of $\psi$ is fixed at its initial postshock value.

For an interface of finite thickness interacting with a series of incident and reflected shocks and expansions, Brouillette $^{13}$ proposed to linearly superpose the effects of each shock wave, taking for the initial condition of one shock the Atwood number, amplitude, and thickness generated by all previous interactions, so that, after $N+1$ interactions, the amplitude is governed in time by

$$
\left(\frac{d \eta}{d t}\right)_{N}=k \sum_{i=0}^{N} \frac{[v]_{i} A_{i}^{\prime} \eta_{i}^{\prime}}{\psi_{i}},
$$

where $[v]_{i}$ is the velocity jump induced on the interface by the $i$ th wave ( $i=0$ corresponding to the incident wave, $i=1$ to the first reflected wave, and so on), $A_{i}^{\prime}$ and $\eta_{i}^{\prime}$ are the Atwood ratio and the amplitude of the perturbations after the passage of the $i$ th wave, and $\psi_{i}$ is the growth reduction factor evaluated from $A_{i}^{\prime}$ and $\delta_{i}^{\prime}$. Brouillette ${ }^{13}$ reported reasonable agreement between his model and his experiments after the interaction of the interface with the first reflected shock and expansion, even into a regime when the small amplitude approximation is no longer satisfied.

\section{B. Interface thickness}

In addition to the amplitude of the perturbations, the other important interface property is its thickness $\delta$, which is determined by the turbulent mixing processes induced by the Richtmyer-Meshkov instability. Most of the analytical and numerical work developed so far has concerned nominally flat interfaces of both zero and finite initial thickness. Perturbations on these interfaces are of the random, small-scale type, and grow very rapidly out of the linear regime. The accepted suggestion is that merging of these small-scale fluctuations results in the formation of large-scale ones (e.g., see Ref. 24). The interface evolves into a 'turbulent mixing zone' (TMZ), whose evolution in time is strongly dependent on the available turbulent kinetic energy, which is a fraction of the total kinetic energy of the interface. In the Richtmyer- 
Meshkov instability, energy is deposited at the interface only by the interaction with the shock wave and any subsequent waves that may arrive at the interface, while viscosity tends to dissipate the turbulent kinetic energy into heat.

\section{Richtmyer-Meshkov induced mixing}

Neglecting viscosity, and assuming independence of initial conditions, dimensional analysis shows that during the initial stages, when no characteristic length scales are present, the evolution of the interface thickness can be described by a relation of the type

$$
\delta=f\left(A^{\prime}\right)[v] t .
$$

Andronov et al. ${ }^{25,26}$ studied the evolution of shock-induced mixing both experimentally and analytically, using turbulence diffusion models. Other experiments were performed by Zaitzev et al. ${ }^{15}$ Barenblatt ${ }^{27}$ has modeled an infinitely thin instantaneous source of vorticity at the interface between two fluids of the same density. The model relies on the Kolmogorov similarity hypothesis which takes the eddy diffusivity and turbulent energy dissipation coefficients to be dependent only on the eddy size and the mean eddy energy; the eddy size in turn is proportional to the external turbulent length scale, a fraction of the layer thickness, $\delta$. Using a diffusion equation for the time evolution of the turbulent energy, the model yields

$$
\delta \propto t^{2 / 3}
$$

in the case of no dissipation, and

$$
\delta \propto t^{\zeta}, \quad \zeta<\frac{2}{3},
$$

if dissipation is taken into account (this last exponent depending on the model chosen for the energy equation).

Mikaelian ${ }^{28}$ used results by Read and Youngs ${ }^{29}$ relative to an interface under constant acceleration as a departure point to derive the time evolution of the thickness of a shockaccelerated interface as

$$
\delta=0.28[v] A^{\prime} t .
$$

Experiments by Brouillette ${ }^{12,14}$ showed poor agreement with Eq. (7). For an interface interacting with a field of reverberating waves, Brouillette ${ }^{12}$ proposed a (dimensional) correlation of the type

$$
\frac{d \delta}{d t}=\chi \frac{A_{0}}{\delta_{0}^{\prime}} \sum_{i=0}^{N-1}\left|[v]_{i}\right|
$$

and found a value $\chi=0.17 \mathrm{~mm}$ for the correlation factor from his experiments.

\section{APPARATUS}

The experiments are performed in a shock tube, which is vertical to take advantage of gravitational stratification in preparing an interface. The cross section is square to provide the parallel walls necessary for any imaging technique to be used in the study of the flow. In the present experiments a shock wave is launched from the top of the shock tube toward the bottom end where a gas interface has been pre-

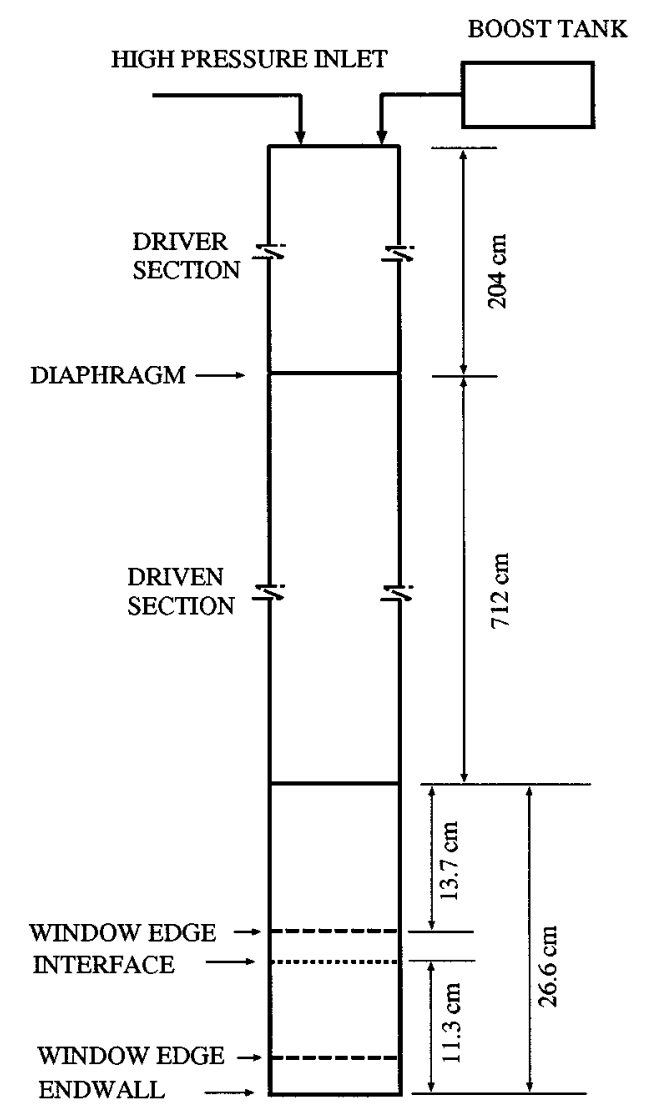

FIG. 1. Schematic of GALCIT vertical square shock tube.

pared. The interface is imaged either by flash $\mathrm{x}$-ray or by schlieren photography. In both cases one image of the interface is obtained during each run.

\section{A. Shock tube}

A schematic of the shock tube is presented in Fig. 1. The driver section is $2.04 \mathrm{~m}$ long, with an inside diameter of 16.5 $\mathrm{cm}$. A detailed description of the shock tube is given by Brouillette. $^{12,30}$

The test section has an 11.4-cm-square inner cross section and is $38.7 \mathrm{~cm}$ long. The interface is initially located within the test section, $11 \mathrm{~cm}$ above the end wall. On one wall of the test section is the slot used to install a plate mechanism for preparing the interface. On the two walls perpendicular to the latter are the openings to mount the windows and their frames. For schlieren imaging, the windows are of optical grade glass, $38 \mathrm{~mm}$ thick. For $\mathrm{x}$ rays, the glass windows are replaced by two carbon fiber plates $1 \mathrm{~mm}$ thick. To support the composite plates against the pressure from the gases inside the test chamber, a pair of dip brazed aluminum grid structures are used. They consist of aluminum plates 1.5 $\mathrm{mm}$ thick and $37 \mathrm{~mm}$ wide, the same as the thickness of the glass windows. They are arranged to form 16 cells: the 4 central ones are square while the remaining 12 are rectangular. The spacing of the grids is different for the two crates to account for the parallax due to conicity $\left(6^{\circ}\right)$ of the $x$-ray beam. Thus, the shadow of the front grid falls on that of the back one, and only one image of the cells is recorded on the film. 


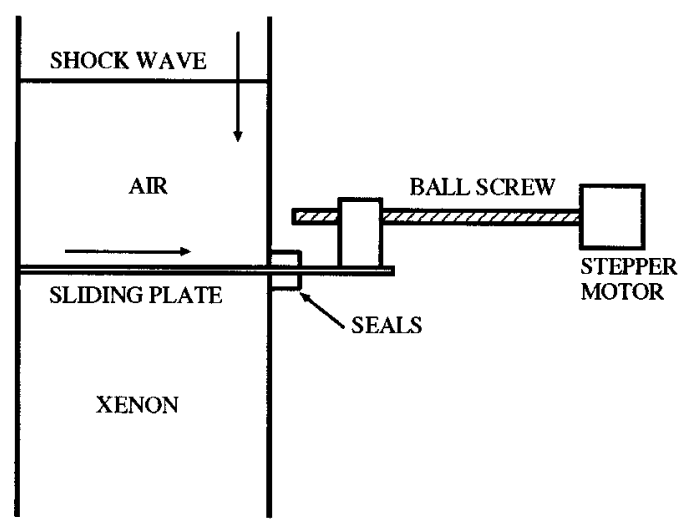

FIG. 2. Plate retraction mechanism.

To prepare an interface between two gases of different densities, the shock tube is equipped with a system designed and built by Brouillette ${ }^{12}$ (see Fig. 2) consisting of a thin (1.2 $\mathrm{mm}$ ) stainless steel plate, a stepper motor to slide the plate in and out of the shock tube, and a plug with a molded silicone rubber gasket to guide the plate in its motion and to seal against leaks from the inside to the outside of the tube.

To prepare the interface, the plate is inserted in the tube, the heavy gas is slowly $(1.5 \mathrm{l} / \mathrm{min})$ introduced into the volume below the plate through an opening at the bottom of the shock tube. As the heavy gas enters, it pushes out the light gas from an opening just beneath the plate, located on the side of the test section. After approximately three times the volume of gas between the retractable plate and the tube bottom has circulated, the gas below the plate is sufficiently pure to perform an experiment.

The plate is retracted at a speed of $10 \mathrm{~cm} / \mathrm{s}$, so that the gases come in direct contact and start to diffuse into each other. During its withdrawal the plate drags along a volume of fluid, due to the no-slip boundary condition on its surface. Once the plate completes its motion out of the test section, the dragged fluid flows back toward the opposite wall and forms surface gravity waves. The waves so generated are principally two-dimensional, but observations of interface perturbations to be discussed below indicate that some threedimensional disturbances may present. Depending on the amount of time, $\tau$, elapsed between the end of plate retraction and shock arrival at the interface, the wave motion results in different initial conditions. These cannot be determined by pictures of the interface taken before its interaction with a shock because the wave amplitude is small and not detectable. However, on the basis of schlieren photographs and $\mathrm{x}$-ray images taken after shock acceleration of the interface when the perturbations have grown, one can draw the following conclusions: For $\tau \approx 1.2 \mathrm{~s}$ the interface is quasisinusoidal across its span; for convenience, this type of interface will be called "single scale" throughout the rest of this work. For $4.0<\tau<8.0 \mathrm{~s}$, the interface is wholly of diffused type, featuring random perturbations of small amplitude; this type of interface will be called "multi-scale" in the rest of this work.

From the results of the x-ray imaging it is deduced that, in some runs, stratification takes place in the xenon during its

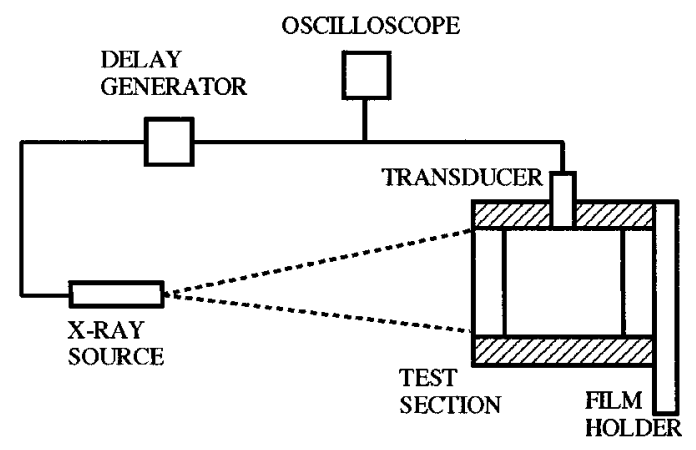

FIG. 3. Layout of x-ray imaging system.

introduction. It is speculated that some leaking occurs along the perimeter of the plate at joints between the windows and the test section, causing some xenon to enter the air volume above the plate, and some air to contaminate the xenon field below it.

\section{B. Gases}

In all of the experiments the driver gas is nitrogen at room temperature. The test gas for the radiography experiments is xenon, chosen because of its large x-ray absorption. ${ }^{31}$ For the schlieren experiments both xenon and sulfur hexafluoride are used.

\section{Imaging techniques}

\section{Radiography}

Figure 3 shows a plan view of the experimental layout. A flash X-ray source (Hewlett-Packard Model 43731 A) is mounted $1.83 \mathrm{~m}$ from the rear window of the test section, with its axis perpendicular to the planes of the windows and to the direction of motion of the plate. The source generates a $50 \mathrm{~ns}$ x-ray flash. During each run a $20 \times 25 \mathrm{~cm}$ x-ray negative (3M Corp., type X-M), sandwiched between two fluorescent screens (3M Corp., type T12) in a film holder mounted on the outside of the rear window, is exposed. A schematic of the film holder and the test section is presented in Fig. 4. A pair of aluminum step wedges (of thickness ranging from $0.3-14.4 \mathrm{~mm}$ ) are used for calibration. They are placed on the front of the film holder, in the area not covered by the test section. Thus, when the x-ray flash is fired, the images of the gases inside the test section and of the aluminum step wedges are recorded on the negative. The radiation from the $\mathrm{x}$-ray source is spatially nonuniform and the intensity varies from shot to shot. Both of these issues are accounted for in the data reduction procedure.

\section{Schlieren system}

The setup consists of a standard Z-folded layout. A spark gap is located at the focus of a spherical mirror (of focal length $1.5 \mathrm{~m}$ and clear aperture $20 \mathrm{~cm}$ ). The diverging light beam is collimated by the mirror and reflected through the test section. An identical mirror collects the parallel beam and focuses it onto a knife edge. The image is recorded on black and white film with a $35 \mathrm{~mm}$ camera, using an $85 \mathrm{~mm}$ 


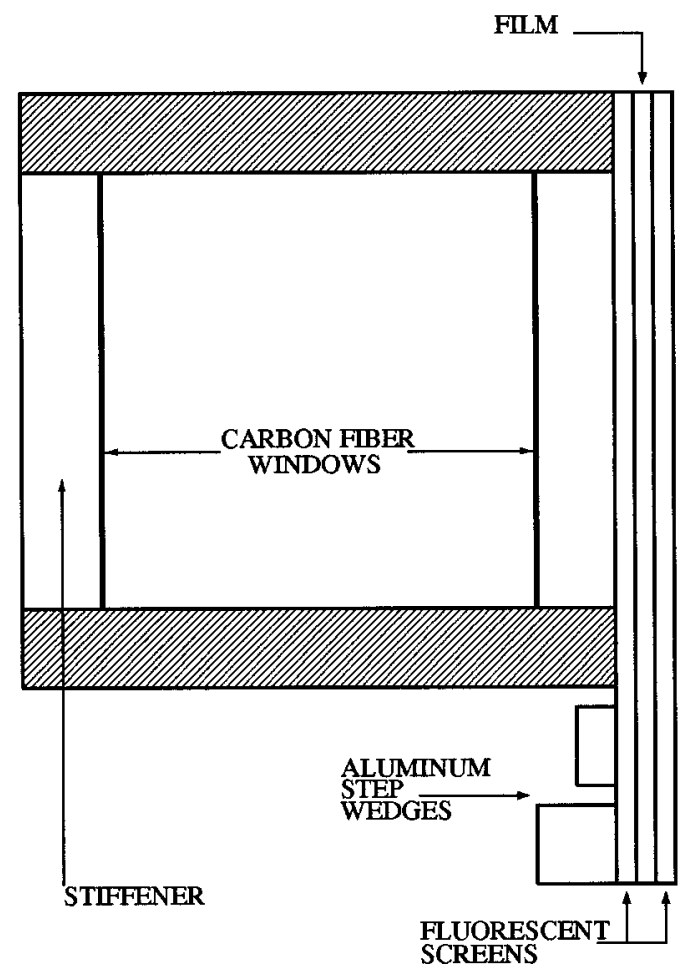

FIG. 4. Detail of test section and film holder.

lens mounted on bellows. To adjust the schlieren sensitivity the position of the knife edge can be varied with a precision $x-y$ positioner.

\section{IMAGE ANALYSIS}

\section{A. Image processing}

The optical density of the developed film is defined by

$$
D \equiv \log \frac{I_{v}}{I_{v 0}},
$$

where $I_{v 0}$ is the intensity of a beam of visible light incident on one side of the negative, and $I_{v}$ is the intensity of the beam of light transmitted through the negative. To measure their optical density, the negatives are illuminated with a Truelite DC blacklight Illumination System and imaged with a Sony AVC-D1 CCD camera whose output is digitized with an Epix Inc. Silicon Video Frame Grabber (installed on an IBM AT microcomputer). The optical density is a monotonic function of the exposure, which in turn is a time integral of the intensity of the $\mathrm{x}$ rays that reach the fluorescent screens between which the film is sandwiched. The intensity is spatially modulated by the xenon (in the test section) and by the aluminum step wedges, following Lambert's law:

$$
\frac{I_{x}}{I_{x 0}}=\exp \left[-\sigma\left(P_{E}\right) \rho l\right],
$$

where $I_{x 0}$ is the intensity of the x-ray beam, of photon energy $P_{E}$, impinging on a thickness $l$ of a material of density $\rho$ and x-ray absorption coefficient $\sigma\left(P_{E}\right)$, and $I_{x}$ is the intensity of the $\mathrm{X}$-ray beam emerging from the slab. In the present case, the aluminum has a spatially constant density and vari- able thickness, as described in Sec. III C 1. The xenon, on the other hand, has constant thickness (the depth of the test section) and density varying in space, $\rho=\rho(x, y, z)$. For this analysis we assume that the $\mathrm{x}$ rays are plane waves.

When the density varies in the $z$ direction, the direction of propagation of the $x$ rays, Lambert's law takes on the form

$$
\frac{I_{x}}{I_{x 0}}=\exp \left(-\sigma\left(P_{E}\right) \int_{0}^{l} \rho(x, y, z) d z\right) .
$$

The term

$$
d=\sigma \int_{0}^{l} \rho d z
$$

is the optical depth of the medium traversed by the $\mathrm{x}$ ray. The measured density is therefore the average across the depth of the test section. X-ray absorption is low in regions of low xenon density and the radiographs appear correspondingly dark (high $D$ ); conversely, x-ray absorption is large in regions of high xenon density and the negatives are more transparent there (low $D$ ). Through an appropriate calibration, one can link the optical density of the film to the local xenon density.

Image processing begins with the digitization of the negatives which yields a field of $376 \times 480$, 8-bit pixel values. In processing this field it is necessary to account and correct for the following items:

(1) temporal fluctuations of the intensity of the illumination box and of the CCD output;

(2) calibration of the CCD response;

(3) spatial nonuniformity of the illumination box;

(4) CCD "pixel noise" (spatial fluctuations);

(5) spatial nonuniformity of the X-ray beam;

(6) relationship between xenon density and aluminum thickness;

(7) temporal variations of the x-ray beam (from shot to shot) both in intensity and spectral composition.

The procedure for analyzing the images is summarized as follows:

(1) Evaluate the optical density field from the CCD output.

(2) Correct the measured optical densities for spatial nonuniformities of the x-ray source.

(3) Develop a calibration curve relating xenon density to aluminum thickness $\rho\left(l_{\mathrm{Al}}\right)$ where $l_{\mathrm{Al}}$ is the aluminum thickness (once, from radiographs of known xenon densities).

(4) Develop a calibration curve relating optical density to aluminum thickness (for each negative, accounting for intensity variation of the x-ray source). For each negative, the averaged optical density of each aluminum step is evaluated and the function

$l_{\mathrm{Al}}(D)=D_{0}\left[\operatorname{csch}\left(D-D_{1}\right)-\operatorname{csch}\left(D_{2}-D_{1}\right)\right]$

is fitted to the data to determine the constants $\left(D_{0}, D_{1}, D_{2}\right)$.

(5) Eliminate the aluminum thickness between the previous two curves, yielding a relation between xenon density and optical density (for each negative). 
(a)

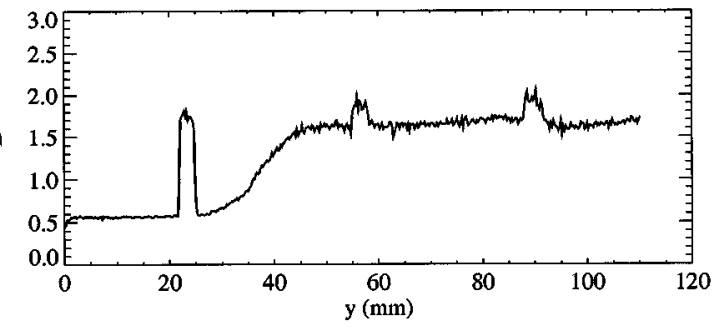

(b)

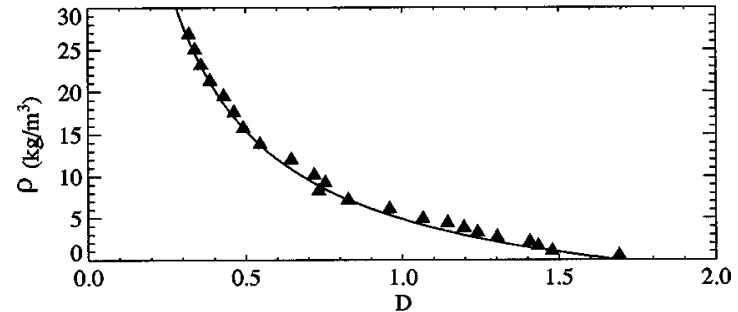

(c)

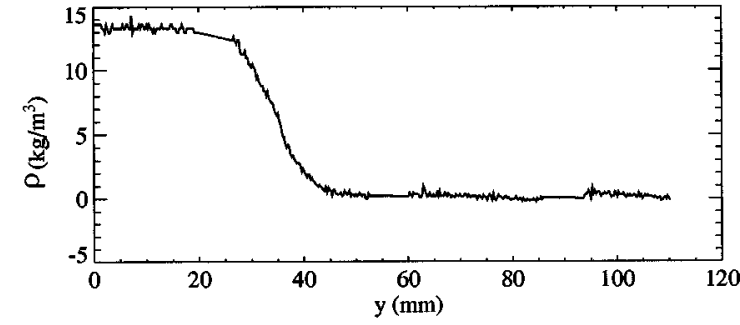

FIG. 5. Calibration steps: (a) optical density profile; (b) calibration curve obtained from [Eq. (13)]; $\boldsymbol{\Delta}$ : gray levels of known xenon densities; nonlinear least squares fit; (c) xenon density profile, $\rho(y)$, at $x=45 \mathrm{~mm}$, obtained eliminating $D$ between (a) and (b). Run \#0547: $\tau=8.0 \mathrm{~s}, M_{i}=1.32$, and $t=1.08 \mathrm{~ms}$.

(6) Apply to (1) above to obtain $\bar{\rho}(x, y)$, where $\bar{\rho}$ is the integrated average (over the depth of the test section) of the xenon density at a point of coordinates $(x, y)$ in the field of view.

An example of the application of this procedure is shown in Fig. 5. A column of pixels at a distance $x$ from the left edge of the field of view is selected. From the profile of corrected optical density across the interface [Fig. 5(a)], through the calibration curve for the negative [Fig. 5(b)], one gets the corresponding xenon density profile [Fig. 5(c)]. This last plot shows that a linear interpolation has been carried out across the horizontal walls of the stiffening structure [corresponding to the three spikes in Fig. 5(a)].

\section{B. Data reduction}

\section{Interface distortion}

A quantitative measure of the distortion of the interface is developed by defining a "mean interface shape" $y_{c}(x)$. Fourier decomposition of $y_{c}(x)$ yields information about the time evolution of the modes. To define a "mean interface shape" a xenon density profile is constructed for each vertical column of pixels in the image. The $y$ coordinate of the centroid of the density profile at each $x$ location is calculated from

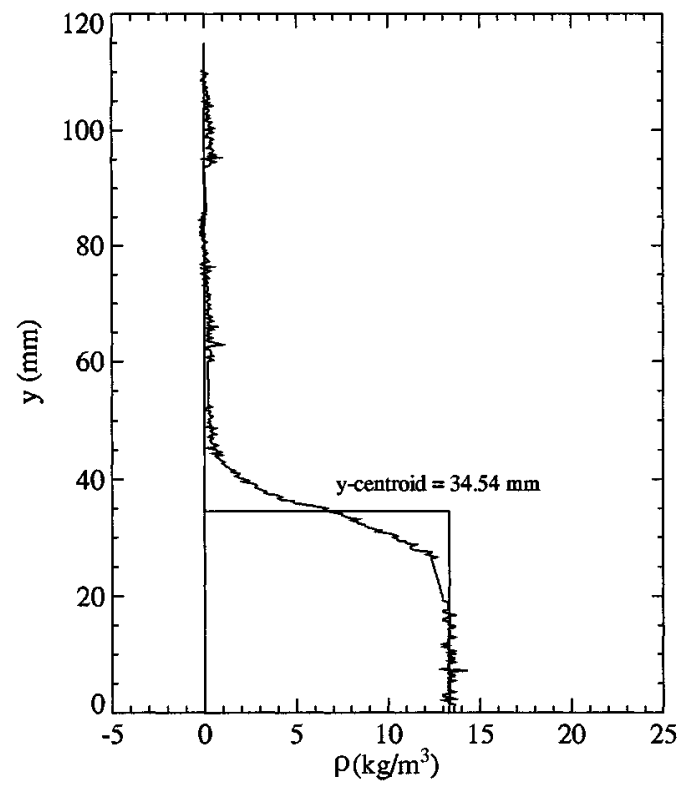

FIG. 6. Determination of the centroid of a density profile (air/Xe). Run $\# 0547: \tau=8.0 \mathrm{~s}, M_{i}=1.32, t=1.08 \mathrm{~ms}$, and $x=17.1 \mathrm{~mm}$.

$$
y_{c}(x)=\frac{\int_{0}^{Y} \rho(x, y) d y}{\rho_{m}}
$$

(see Fig. 6), where $Y$ is the height of the field of view in the test section and $\rho_{m}$ is the asymptote in the xenon field. The locus of the centroids is the mean interface shape $y_{c}(x)$. The shape constructed by Eq. (14) exhibits high-frequency noise (partly due to the CCD noise unsuppressed by averaging over 25 digitizations, and partly to the graininess of the film) and three gaps corresponding to the vertical elements of the stiffening grid. The gaps are filled by linear interpolation. To carry out a modal analysis of $y_{c}(x)$, its spatial spectrum is constructed by FFT. To minimize the undesired effects of truncation at the edges of the image, the function $y_{c}(x)$ is windowed with a Hanning function, given by

$$
H(x)=\frac{1}{2}\left[1-\cos \left(\frac{2 \pi x}{L}\right)\right],
$$

where $L$ is the width of the field of view. To generate a smooth interface shape (for plotting purposes), the FFT of $y_{c}(x)$ is taken without the use of the Hanning window, and the shape is reconstructed using only the first six modes of the spectrum (a low-pass digital filter in the frequency domain). An example of a smoothed shape is compared to the unsmoothed version in Fig. 7. Partial reconstruction without windowing suffers from truncation effects, as can be seen at the extreme edges.

\section{Interface thickness}

To determine the interface thickness from the density data, one needs a density profile, $\bar{\rho}(y)$, free of any artificial thickening introduced by averaging over the distortion of the interface. In the extreme case, obtaining a density profile of a discontinuous, sinusoidal interface, by averaging across its entire span, does not give a step function but a curve with a finite gradient, and the interface thickness deduced from this 


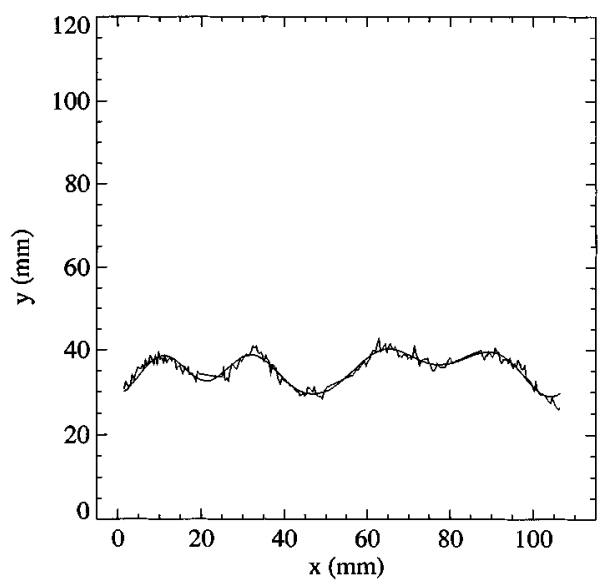

FIG. 7. Mean interface shape. Air/Xe. Run \#0516: $\tau=1.2 \mathrm{~s}, M_{i}=1.32$, and $t=5.85 \mathrm{~ms}$

curve coincides with the amplitude of the sinusoid. To avoid this, before averaging, the profile at each $x$ location is shifted vertically by the distance $y_{c}(x)$. This makes the interface flat. A typical result of this operation, on the data with interface distortion given in Fig. 7, is the density profile shown in Fig. 8.

The interface thickness, $\delta$, is defined by (see Fig. 8)

$$
\delta=h_{1}+h_{2},
$$

where

$$
h_{1}=\frac{1}{\rho_{c}} \int_{y_{c}}^{Y} \rho d y,
$$

and

$$
h_{2}=\frac{1}{\rho_{m}-\rho_{c}}\left(\rho_{m} y_{c}-\int_{0}^{y_{c}} \rho d y\right) .
$$

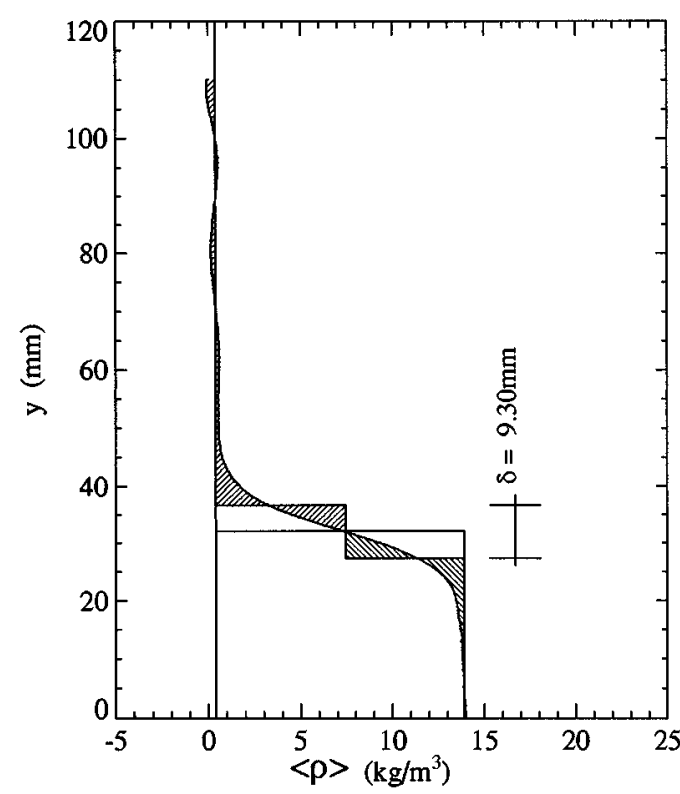

FIG. 8. Average density profile and interface thickness. Air/Xe. Run \#0547, $\tau=8.0 \mathrm{~s}, M_{i}=1.32$, and $t=1.08 \mathrm{~ms} ; x_{l}=0$ and $x_{r}=105$.

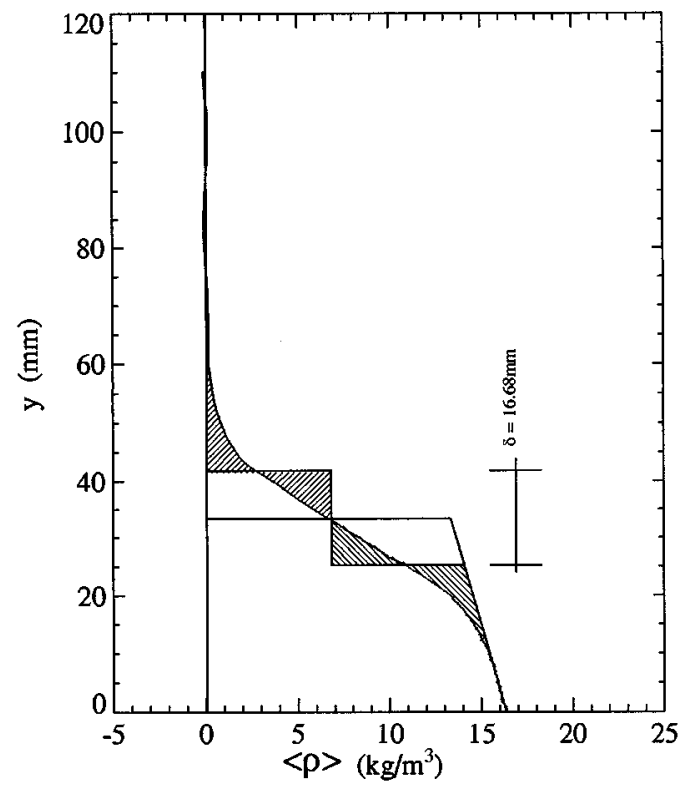

FIG. 9. Average density profile and interface thickness for nonuniform xenon density asymptote. Air/Xe. Run \#0533: $\tau=1.2 \mathrm{~s}, M_{i}=1.32$, and $t=5.57$ $\mathrm{ms} ; x_{l}=0$ and $x_{r}=105$.

The construction of this integral definition of thickness is indicated in Fig. 8.

When the xenon is stratified so that the asymptote is nonuniform, a trapezoid is used instead of a rectangle, as shown in Fig. 9. The thicknesses obtained from profiles with nonuniform asymptotes are indicated with circles in the figures of Sec. V.

\section{Density contour plots}

Another way to examine the data is to generate contour plots of the density field. For this purpose, the density values

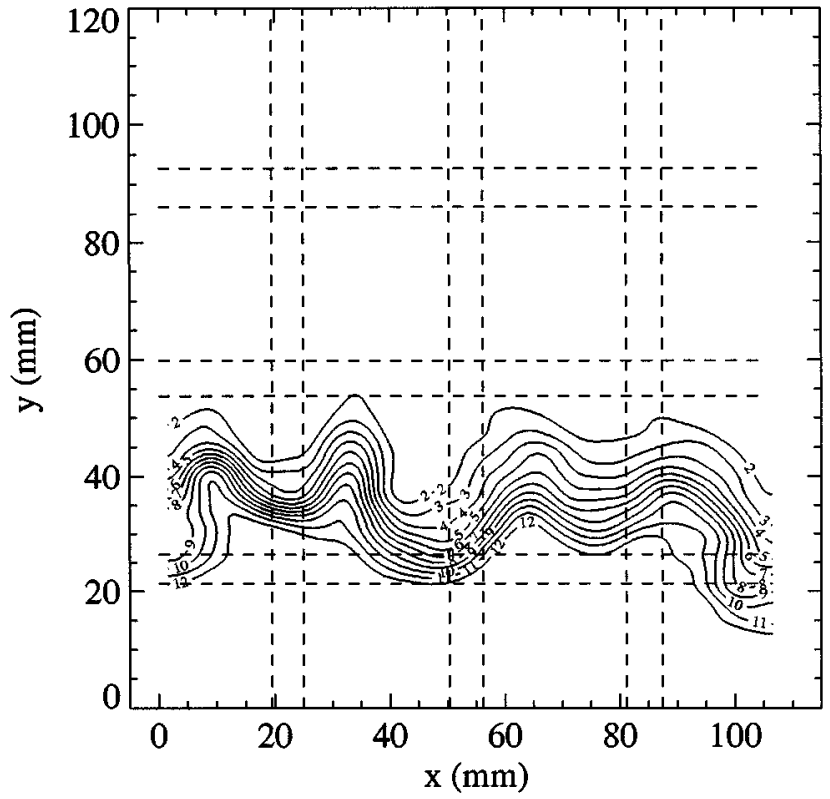

FIG. 10. Density contours generated from filtered two-dimensional density field. Air/Xe. Run \#0516: $\tau=1.2 \mathrm{~s}, M_{i}=1.32$, and $t=5.85 \mathrm{~ms}$. 


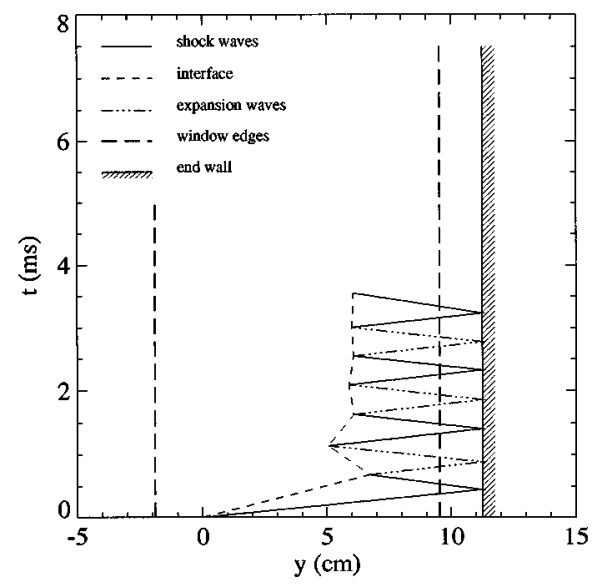

FIG. 11. The $y-t$ diagram for the close end wall configuration (air/Xe, $M_{i}=1.32$ ).

are linearly interpolated across the regions corresponding to the walls of the stiffening structure. The field is then extended by constructing its mirror image with respect to the $x$ and $y$ axes. The 2-D FFT of this field is taken and multiplied by an ideal low-pass filter, consisting of a cylinder of height 1 and elliptical cross section. The two semiaxes correspond to the number of modes used in the $x$ and $y$ directions, respectively. An example of a smoothed density field, generated with a filter having $20 \times 40$ modes, is shown in Fig. 10 . The dashed lines in the figure indicate regions across which the data are interpolated.

\section{EXPERIMENTS}

The objective is to study the effect of multiple wave reverberations on the growth of the interface perturbations and thickness. Both pseudo-single scale and multi-scale interfaces are studied, using incident shock waves of $M_{i}=1.32$ and $M_{i}=1.52$.

As an example, the $y-t$ diagram for an air/xenon interface accelerated by a $M_{i}=1.32$ shock wave is shown in Fig. 11. The origins of the $y$ and $t$ axes coincide with the initial position of the interface and the arrival of the incident shock, respectively. The two dashed, vertical lines represent the edges of the test section window. The incident shock sets the interface in motion toward the bottom of the shock tube. For $M_{i}=1.32$, the Mach number of the shock transmitted in the xenon is $M_{t}=1.44$, while that of the shock reflected in the air is $M_{r_{0}}=1.10$. The transmitted shock reflects from the end wall of the shock tube with Mach number $M_{r_{1}}=1.36$ intercepts the downward moving interface at time $t_{c}$ and, since

TABLE I. Interaction times and interface positions. $t_{s}$ : time of interaction with first reflected shock. $y_{s}$ : position of the interface at time $t_{s} . t_{e}$ : time of interaction with first reflected expansion. $y_{e}$ : position of the interface at time $t_{e}$. Distances are measured from the initial position of the interface.

\begin{tabular}{lcccc}
\hline \hline \multicolumn{1}{c}{ Conditions } & $t_{s}(\mathrm{~ms})$ & $y_{s}(\mathrm{~mm})$ & $t_{e}(\mathrm{~ms})$ & $y_{e}(\mathrm{~mm})$ \\
\hline Air/Xe, $M_{i}=1.32$ & 0.68 & 68 & 1.16 & 53 \\
Air $/ \mathrm{Xe}, M_{i}=1.52$ & 0.54 & 79 & 0.81 & 60 \\
Air $/ \mathrm{SF}_{6}, M_{i}=1.32$ & 0.83 & 88 & 1.39 & 68 \\
\hline \hline
\end{tabular}

TABLE II. Relevant parameters for $M_{i}=1.32$, air/Xe interface. $v$ : vertical component of the velocity. $\rho$ : density. $a$ : speed of sound. A: Atwood number. The arrows indicate the direction of the mean interface motion.

\begin{tabular}{llcccc}
\hline \hline & & Preshock & Shock & Reshock & Expansion \\
\hline \multirow{4}{*}{ Air } & $v(\mathrm{~m} / \mathrm{s})$ & 0 & $98 \downarrow$ & $37 \uparrow$ & $12 \downarrow$ \\
& $\rho\left(\mathrm{kg} / \mathrm{m}^{3}\right)$ & 1.21 & 2.19 & 3.06 & 2.65 \\
& $a(\mathrm{~m} / \mathrm{s})$ & 340 & 389 & 416 & 405 \\
$\mathrm{Xe}$ & $\rho a\left(\mathrm{~kg} / \mathrm{m}^{2} \mathrm{~s}\right)$ & 411 & 852 & 1273 & 1073 \\
& $\rho\left(\mathrm{kg} / \mathrm{m}^{3}\right)$ & 5.46 & 8.90 & 11.65 & 10.18 \\
& $a(\mathrm{~m} / \mathrm{s})$ & 176 & 211 & 233 & 227 \\
& $\rho a\left(\mathrm{~kg} / \mathrm{m}^{2} \mathrm{~s}\right)$ & 960 & 1878 & 2715 & 2311 \\
& $(\rho a)_{\mathrm{Xe}}$ & 2.33 & 2.22 & 2.13 & 2.15 \\
\cline { 2 - 2 } & $(\rho a)_{\mathrm{Air}}$ & 0.637 & 0.605 & 0.584 & 0.587 \\
\hline \hline
\end{tabular}

the xenon's acoustic impedance is higher than the air's, a shock is transmitted and a rarefaction is reflected; the interface is set in an upward motion. The rarefaction reaches the bottom of the shock tube, reflects and catches up with the upward moving interface at time $t_{e}$; it is reflected as a compression wave and transmitted as a rarefaction. These reverberations continue in time, following the same pattern. For $M_{i}=1.52$, the shock reflected in the air has $M_{r_{0}}=1.15$, the shock transmitted in the xenon has $M_{t}=1.73$; the transmitted shock reflects from the end wall with $M_{r_{1}}=1.53$. For an air $/ \mathrm{SF}_{6}$ interface and a $M_{i}=1.32$ shock the transmitted wave has $M_{t}=1.49$, and the shock reflected in the air has $M_{r_{0}}$ $=1.09 ;$ the wave reflected from the end wall has $M_{r_{1}}$ $=1.47$.

The interaction times $t_{c}$ and $t_{e}$, and interface position $y_{p}$ for the three cases are given in Table I. The interface velocity $v$, the density $\rho$, speed of sound $a$, and acoustic impedance $\rho a$ of the two gases upon the first two wave interactions are reported in Tables II-IV (the arrows indicate the direction in which the interface is moving). All the data presented in these three tables are calculated using one-dimensional gasdynamics.

\section{A. Single-scale interfaces \\ 1. X-ray imaging and densitometry}

Figure 12 is the digital image of the optical density field $0.26 \mathrm{~ms}$ after the incident shock has interacted with an airxenon interface. The image is 8 bits deep, with gray scale

TABLE III. Relevant parameters for $M_{i}=1.52$, air/Xe interface. $v$ : vertical component of the velocity. $\rho$ : density. $a$ : speed of sound. A: Atwood number. The arrows indicate the direction of the mean interface motion.

\begin{tabular}{llcccc}
\hline \hline & & Preshock & Shock & Reshock & Expansion \\
\hline \multirow{4}{*}{ Air } & $v(\mathrm{~m} / \mathrm{s})$ & 0 & $152 \downarrow$ & $55 \uparrow$ & $25 \downarrow$ \\
& $\rho\left(\mathrm{kg} / \mathrm{m}^{3}\right)$ & 1.21 & 2.86 & 4.56 & 3.82 \\
& $a(\mathrm{~m} / \mathrm{s})$ & 340 & 417 & 460 & 451 \\
$\mathrm{Xe}$ & $\rho a\left(\mathrm{~kg} / \mathrm{m}^{2} \mathrm{~s}\right)$ & 411 & 1193 & 2098 & 1723 \\
& $\rho\left(\mathrm{kg} / \mathrm{m}^{3}\right)$ & 5.46 & 10.82 & 15.70 & 13.78 \\
& $a(\mathrm{~m} / \mathrm{s})$ & 176 & 233 & 271 & 260 \\
& $\rho a\left(\mathrm{~kg} / \mathrm{m}^{2} \mathrm{~s}\right)$ & 960 & 2521 & 4255 & 3583 \\
& $(\rho a)_{\mathrm{Xe}}$ & 2.33 & 2.11 & 2.03 & 2.08 \\
\cline { 2 - 3 } & $(\rho a)_{\mathrm{Air}}$ & 0.637 & 0.582 & 0.550 & 0.566 \\
$A$ & & & & & \\
\hline \hline
\end{tabular}


TABLE IV. Relevant parameters for $M_{i}=1.32$, air/SF ${ }_{6}$ interface. $v$ : vertical component of the velocity. $\rho$ : density. $a$ : speed of sound. A: Atwood number. The arrows indicate the direction of the mean interface motion.

\begin{tabular}{llcccc}
\hline \hline & & Preshock & Shock & Reshock & Expansion \\
\hline \multirow{4}{*}{ Air } & $v(\mathrm{~m} / \mathrm{s})$ & 0 & $106 \downarrow$ & $38 \uparrow$ & $22 \downarrow$ \\
& $\rho\left(\mathrm{kg} / \mathrm{m}^{3}\right)$ & 1.21 & 2.15 & 3.08 & 2.94 \\
& $a(\mathrm{~m} / \mathrm{s})$ & 340 & 417 & 460 & 413 \\
$\mathrm{Xe}$ & $\rho a\left(\mathrm{~kg} / \mathrm{m}^{2} \mathrm{~s}\right)$ & 411 & 832 & 1284 & 1216 \\
& $\rho\left(\mathrm{kg} / \mathrm{m}^{3}\right)$ & 6.07 & 12.89 & 20.28 & 19.17 \\
& $a(\mathrm{~m} / \mathrm{s})$ & 135 & 140 & 144 & 143 \\
& $\rho a\left(\mathrm{~kg} / \mathrm{m}^{2} \mathrm{~s}\right)$ & 820 & 1805 & 2920 & 2740 \\
& $(\rho a)_{\mathrm{Xe}}$ & 1.99 & 2.17 & 2.27 & 2.25 \\
\cline { 2 - 3 } & $(\rho a)_{\mathrm{Air}}$ & 0.667 & 0.714 & 0.736 & 0.734 \\
\hline \hline & & & & &
\end{tabular}

levels equal to $100 \times D$. The gray scale actually displayed (shown to the side of the test section) has been stretched to enhance the contrast of the negative, by representing the minimum value of the optical density in the negative $(D$ $=0.55$, pixel value 55 , in the xenon region) by $D=0$ and the maximum value $(D=1.65$, pixel value 165 , in the air region) by $D=2.55$. The grid pattern in the picture is the shadow of the aluminum stiffening structure. From top to bottom one can see shocked air (light gray, near white, indicating large $D$ values, large exposure, low x-ray absorption), the interface (the steep transition from the light to the dark gray), shocked xenon (dark gray, indicating small $D$, small exposure, large $\mathrm{x}$-ray absorption), the transmitted shock wave (the sharp discontinuity between dark and medium gray), and unshocked xenon (medium gray, indicating medium $D$, medium exposure, medium x-ray absorption). The interface looks essentially flat; no distortions can be detected by visual inspection of the image. However, as image processing of this image and the subsequent development of the interface will show, perturbations of finite amplitude, and wavelength between 27.5 and $110 \mathrm{~mm}$ (the first four modes) are present. The transmitted shock wave also appears undistorted. Figure 13 shows the average density profile for the run. The top, center, and bottom portions of the curve (essentially vertical) represent the air, shocked, and unshocked xenon fields, respectively. The portion of the curve joining the top and middle

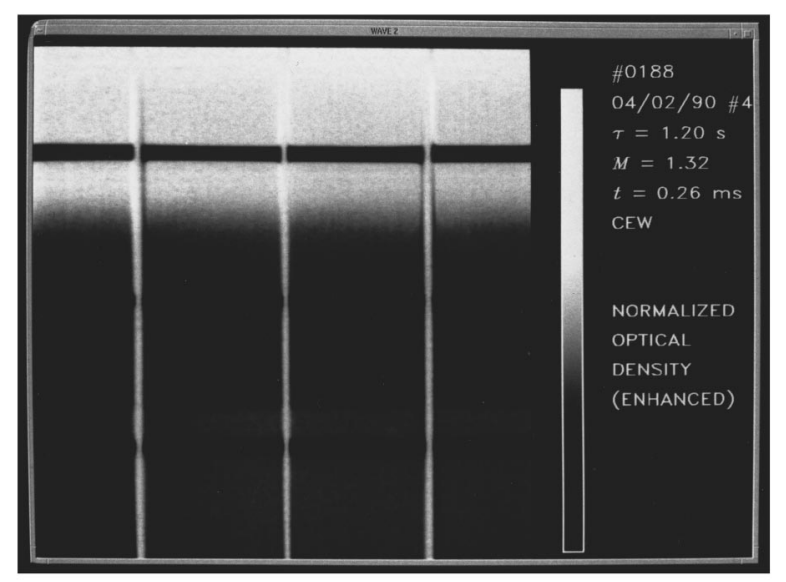

FIG. 12. Air/Xe. Run \#0188: $\tau=1.2 \mathrm{~s}, M_{i}=1.32$, and $t=0.26 \mathrm{~ms}$. Optical density of the radiograph.

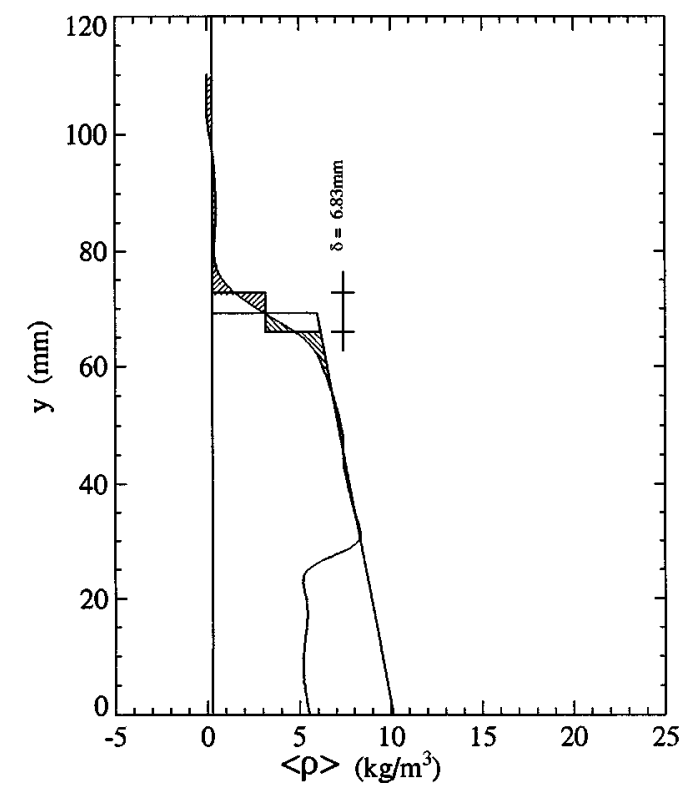

FIG. 13. Air/Xe. Run \#0188: $\tau=1.2 \mathrm{~s}, M_{i}=1.32$, and $t=0.26 \mathrm{~ms}$. Average density profile; $x_{l}=0$ and $x_{r}=105$.

regions represents the interface, while that joining the middle and bottom regions represents the shock wave. The fact that the shock wave does not look like a perfectly horizontal segment in the density profile is a consequence of averaging over a total of about 220 vertical density profiles, following the procedure described in Sec. IV B 2. The interface thickness measured from the average density profile is about 7 $\mathrm{mm}$, but a fraction of this value (up to $40 \%$ ) may be due to the vertical shifting of the individual density profiles, prior to averaging, just like the finite thickness of the shock wave is. No major changes occur on the interface until the interaction with the shock reflected from the end wall. This interaction takes place at $t=0.68 \mathrm{~ms}$ for $M_{i}=1.32$; then the expansion fan reflected from the bottom reaches the interface at $t=1.16$ ms. In the time interval between these two events the interface does not change its appearance.

At $t=2.04 \mathrm{~ms}, 0.94 \mathrm{~ms}$ after interaction with the reflected expansion, the interface clearly shows three troughs and four crests (Fig. 14). At the sides of the interface, two circular low-density regions, trapped between the interface and the walls, are visible. They are wall vortices caused by the interaction of the reflected shocks with the boundary layer generated by the incident shock. By this time, the interface is stationary about $33 \mathrm{~mm}$ above the lower edge of the test section window.

By $t=5.85 \mathrm{~ms}$ (Fig. 15) the crest amplitude is of the order of the wavelength of the perturbations. Mushroom-like distortions on the sides of the second crest from the left can be seen in the original radiograph. By this time the difference between the distortion of the upper and lower layers of the interface has become substantial (Fig. 16). Because of its integral definition, the interface shape plotted in Fig. 7 represents a mean distortion somewhere in between that of the top and bottom layers. At even later times, the integral averaging actually results in an apparent flattening of the mean interface shape. 


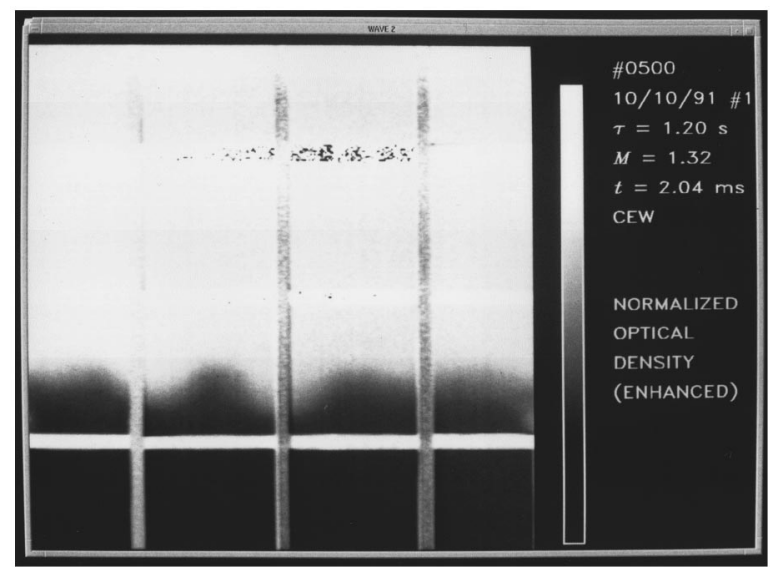

FIG. 14. Air/Xe. Run \#0500: $\tau=1.2 \mathrm{~s}, M_{i}=1.32$, and $t=2.04 \mathrm{~ms}$. Optical density of the radiograph.

The evolution of the amplitude of the modes of the disturbances is shown in Figs. 17 and 18. Open symbols represent data adversely affected by the flattening described above. The three vertical lines in each of the six plots indicate the arrival at the interface of the first reflected shock, first reflected expansion, and second reshock, respectively. Values for linear growth rates are obtained by least squares interpolation over different time intervals. Expressions for the linear fits appear in the plots for modes 1-4, with the amplitude $\eta$ in $\mathrm{mm}$ and the time $t$ in $\mathrm{ms}$. The inequalities indicate the time range of validity of the linear fits. For $M_{i}=1.52$, the first three modes exhibit growth rates that are approximately twice the corresponding ones in the $M_{i}=1.32$ case, and mode 4 grows about three times faster; for both Mach numbers, all modes show saturation at values of $\eta k$ between $4.3 \times 10^{-3}$ and $2.2 \times 10^{-2}$, except for mode 2 at $M_{i}=1.32$, which seems not to saturate (see Table VI). The time evolution of modes 5 and 6 is also presented, but no linear fit is made to the data since their contribution to the interface shape appears negligible. The measured growth rates are summarized in Table $\mathrm{V}$, where the ranges of validity are indicated as $t_{1}-t_{2}$. Growth rates calculated from the impulsive model, Eq. (3), using the superposition of the ef-

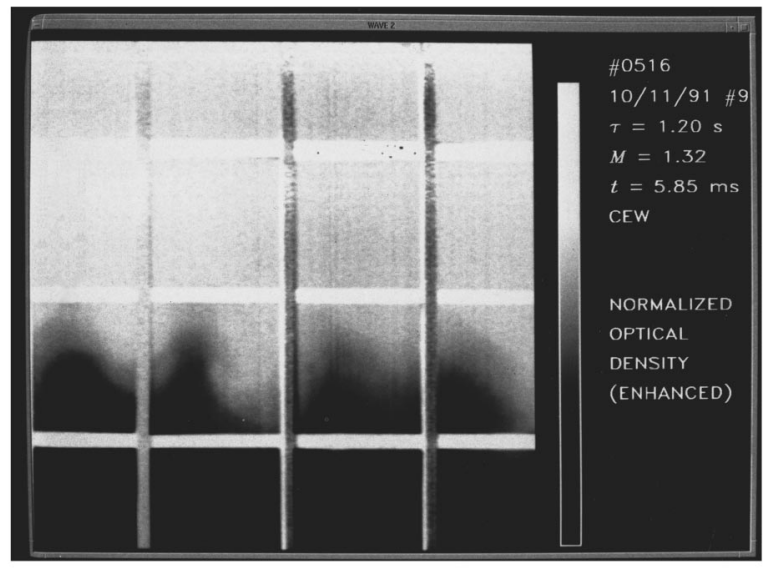

FIG. 15. Air/Xe. Run \#0516: $\tau=1.2 \mathrm{~s}, M_{i}=1.32$, and $t=5.85 \mathrm{~ms}$. Optical density of the radiograph.

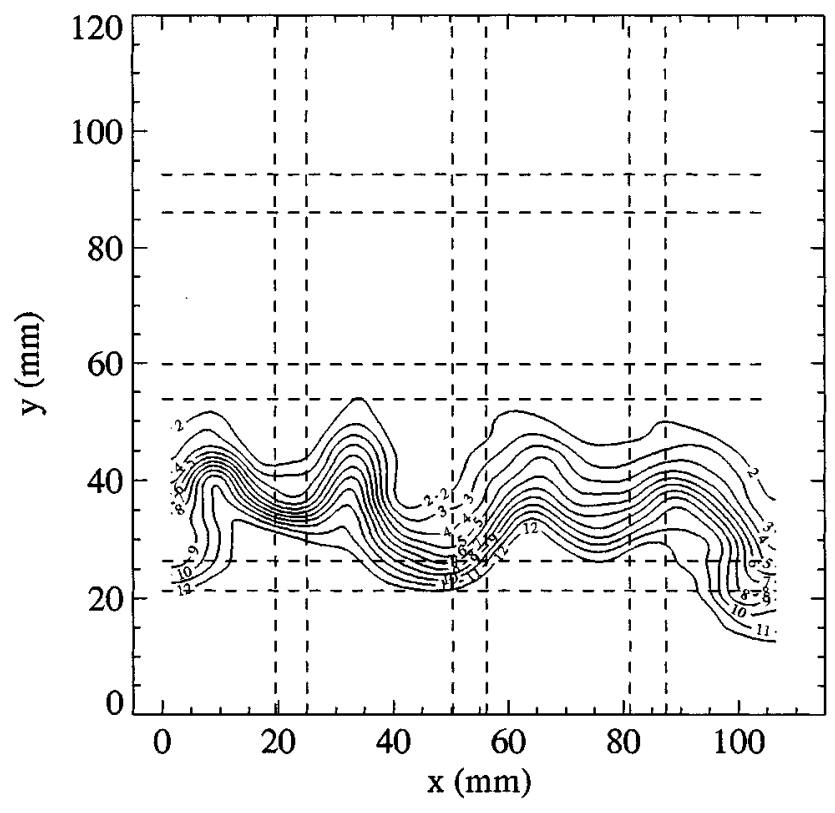

FIG. 16. Air/Xe. Run \#0516: $\tau=1.2 \mathrm{~s}, M_{i}=1.32$, and $t=5.85 \mathrm{~ms}$. Density contours.

fects of the first incident and reflected shocks, first reflected rarefaction, and second reshock, are also presented in Table $\mathrm{V}$. The amplitudes and the thicknesses used to calculate the growth reduction factor $\psi$ are those measured just after the incident shock, the reshock, and the reflected expansion. The saturation values of $\eta k$ are reported in Table VI.
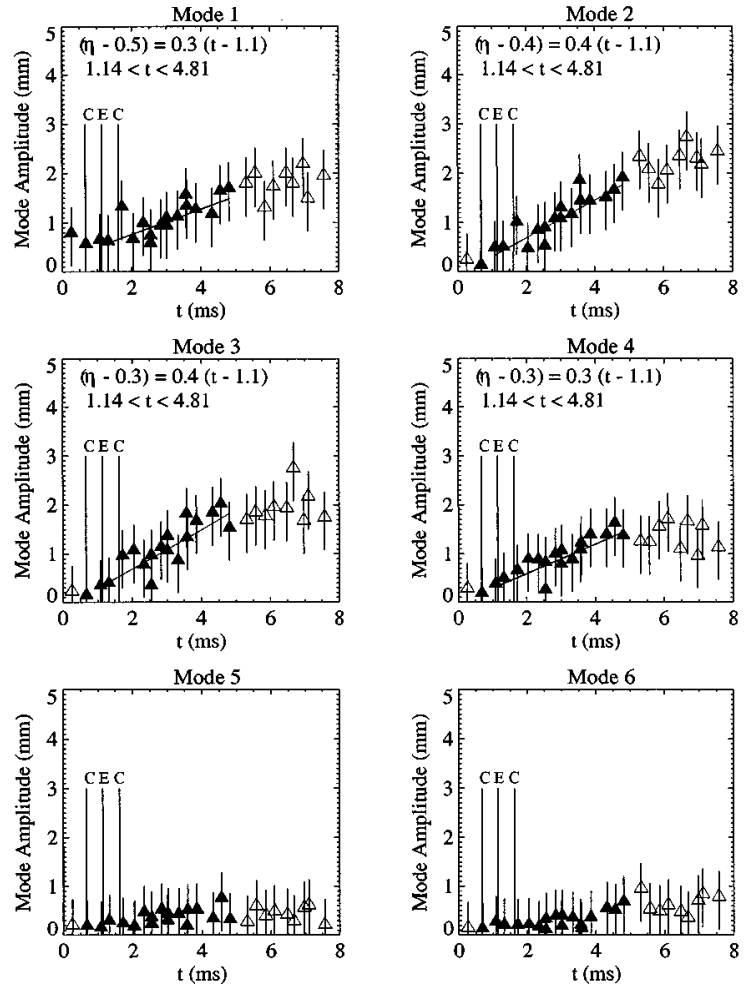

FIG. 17. Air/Xe: $M_{i}=1.32$ and $\tau=1.2 \mathrm{~s}$. Amplitude of the first six modes versus time. Measurements from radiographs: $\boldsymbol{\Delta}$ : data not affected by interface flattening; $\triangle$ : data adversely affected by interface flattening; —: linear least-squares fit. 

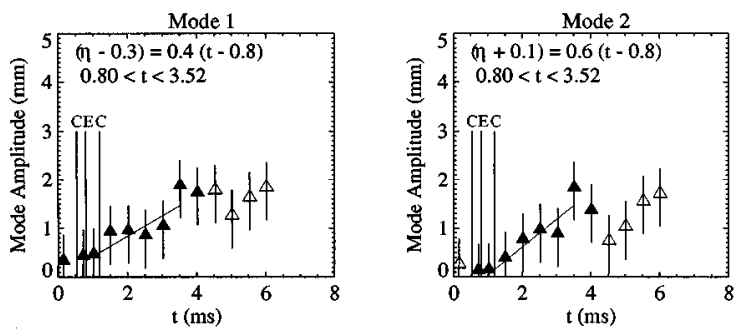

Mode 3

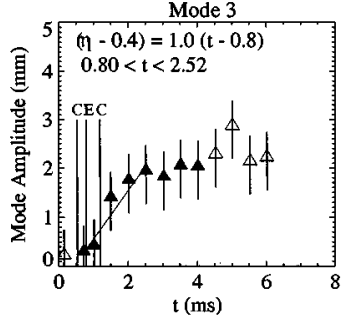

Mode 4

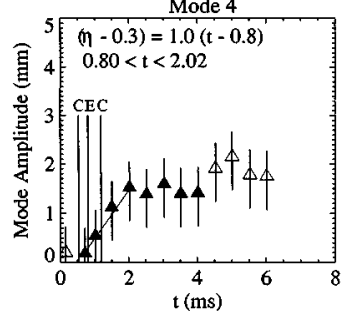

Mode 5
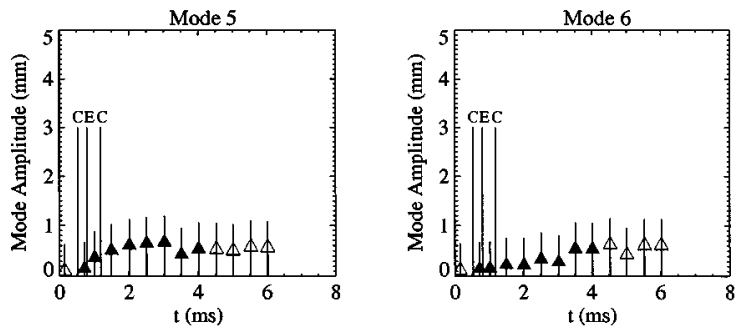

FIG. 18. Air/Xe: $M_{i}=1.52$ and $\tau=1.2 \mathrm{~s}$. Amplitude of the first six modes versus time. Measurements from radiographs: $\boldsymbol{\Delta}$ : data not affected by interface flattening; $\triangle$ : data adversely affected by interface flattening; — : linear least-squares fit.

Other than for modes 1 and 3 with $M_{i}=1.52$, all measured growth rates are smaller than the calculated ones. This is expected for perturbations whose amplitude is of the same order of magnitude of the wavelength and which are therefore no longer in their linear regime. Further reason for the discrepancy between the calculated and measured growth rates comes from the fact that the predicted growth rates depend directly on the values of the amplitudes measured just after each interaction. In the present case the error in these measurements is probably large compared to the value itself, which is small. The measurements of the thickness also have an effect, since the growth reduction factor depends on them.

TABLE V. Growth rates of the first four modes for single-scale interfaces; $t_{1}-t_{2}$ indicates the time range of the linear fit to the data.

\begin{tabular}{|c|c|c|c|c|}
\hline $\begin{array}{l}\text { Mach } \\
\text { No. }\end{array}$ & Mode & $t_{1}-t_{2}(\mathrm{~ms})$ & $\begin{array}{l}\frac{d \eta}{d t}(\mathrm{~m} / \mathrm{s}) \\
\text { measured }\end{array}$ & $\begin{array}{c}\frac{d \eta}{d t}(\mathrm{~m} / \mathrm{s}) \\
\text { calculated }[\mathrm{Eq} .(3)]\end{array}$ \\
\hline \multirow[t]{4}{*}{1.32} & 1 & $1.1-4.8$ & 0.25 & 0.58 \\
\hline & 2 & $1.1-4.8$ & 0.35 & 1.61 \\
\hline & 3 & $1.1-4.8$ & 0.41 & 1.58 \\
\hline & 4 & $1.1-4.8$ & 0.29 & 2.02 \\
\hline \multirow[t]{4}{*}{1.52} & 1 & $0.8-3.5$ & 0.41 & 0.20 \\
\hline & 2 & $0.8-3.5$ & 0.57 & 1.00 \\
\hline & 3 & $0.8-2.5$ & 0.98 & 0.23 \\
\hline & 4 & $0.8-2.0$ & 0.98 & 4.43 \\
\hline
\end{tabular}

TABLE VI. Modal amplitudes at saturation for single-scale interfaces.

\begin{tabular}{cclc}
\hline \hline & & \multicolumn{2}{c}{$\eta k$} \\
\cline { 3 - 4 } Mode & $k\left(\mathrm{~mm}^{-1}\right)$ & \multicolumn{1}{c}{$M_{i}=1.32$} & $M_{i}=1.52$ \\
\hline 1 & $29 \times 10^{-3}$ & $4.9 \times 10^{-3}$ & $4.3 \times 10^{-3}$ \\
2 & $58 \times 10^{-3}$ & No saturation & $7.3 \times 10^{-3}$ \\
3 & $87 \times 10^{-3}$ & $1.7 \times 10^{-2}$ & $1.7 \times 10^{-2}$ \\
4 & $12 \times 10^{-2}$ & $1.7 \times 10^{-2}$ & $2.2 \times 10^{-2}$ \\
\hline \hline
\end{tabular}

Plots of the interface thickness versus time are presented in Fig. 19 for $M_{i}=1.32$ and Fig. 20 for $M_{i}=1.52$, respectively. The triangles indicate that the thickness was measured from a density profile with uniform xenon density asymptote; the circles indicate nonuniform xenon density asymptotes. Open symbols have the same significance as in the plots of modal amplitude versus time. Linear fits made to the postexpansion data indicate that the interface thickness grows at rates of about 1.7 and $1.9 \mathrm{~m} / \mathrm{s}$, respectively, from post-expansion values of about 6 and $5 \mathrm{~mm}$. For both Mach numbers the thickness increases steadily until about $t=5.5$ ms after which it appears to drop (although only one datum is available at late times for the $M_{i}=1.52$ case). The apparent drop may be in part due to the occurrence of the nonuniform asymptote. Over the duration of these experiments no departure from linear behavior can be detected. Comparison with previous experiments is not possible for this case, since this is the first series of thickness measurements on an interface with large wavelength perturbations.

In the absence of shock-induced mixing, laminar molecular diffusion would have caused the interface thickness to grow by less than $1.1 \mathrm{~mm}$ over a time interval of $5 \mathrm{~ms}$. Shock-induced mixing, on the other hand, causes the interface thickness to grow by about $8.5 \mathrm{~mm}$ over the same time, and thus overcomes any thinning that the stretching caused by the growth of the large wavelength perturbations may induce. Using the thickness to quantify the amount of mixing that occurs at the interface, it is seen that the shock-induced turbulence in the interface enhances this mixing by a factor of about 8 .

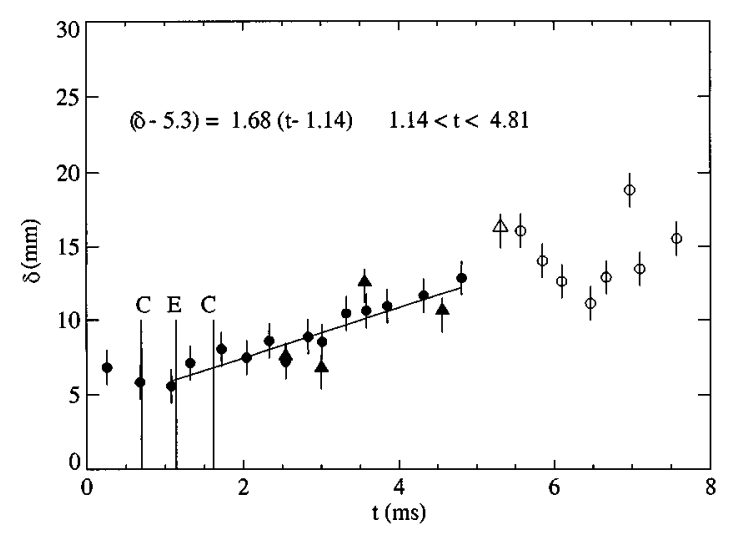

FIG. 19. Air/Xe: $M_{i}=1.32$ and $\tau=1.2$ s. Interface thickness versus time. Measurements from radiographs: $\boldsymbol{\Lambda}, \triangle$ : uniform Xe-density asymptote; $\boldsymbol{\bullet}, \bigcirc$ : non-uniform Xe-density asymptote; $\boldsymbol{\Lambda}, \mathbf{0}$ : data not affected by interface flattening; $\triangle, \bigcirc$ : data adversely affected by interface flattening; - : linear least-squares fit. 


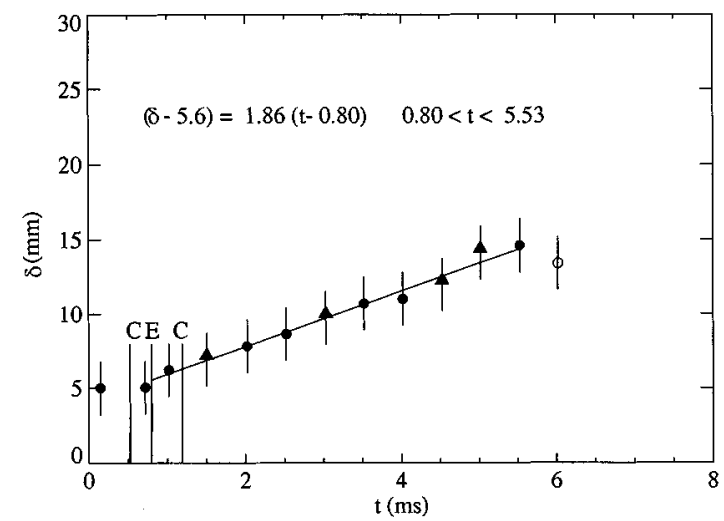

FIG. 20. Air/Xe: $M_{i}=1.52$ and $\tau=1.2 \mathrm{~s}$. Interface thickness versus time. Measurements from radiographs: $\boldsymbol{\Lambda}, \triangle$ : uniform Xe-density asymptote; $\boldsymbol{\bullet}, \bigcirc$ : non-uniform Xe-density asymptote; $\boldsymbol{\Lambda}, \mathbf{0}$ : data not affected by interface flattening; $\triangle, \bigcirc$ : data adversely affected by interface flattening; —: linear least-squares fit.

\section{Schlieren visualization}

Figure 21 shows schlieren photographs of an air $/ \mathrm{SF}_{6}$ interface at $t=2.11 \mathrm{~ms}$ and of an air $/$ xenon one at $t=3.70 \mathrm{~ms}$. The periphery of the second crest from the left, in both pic-

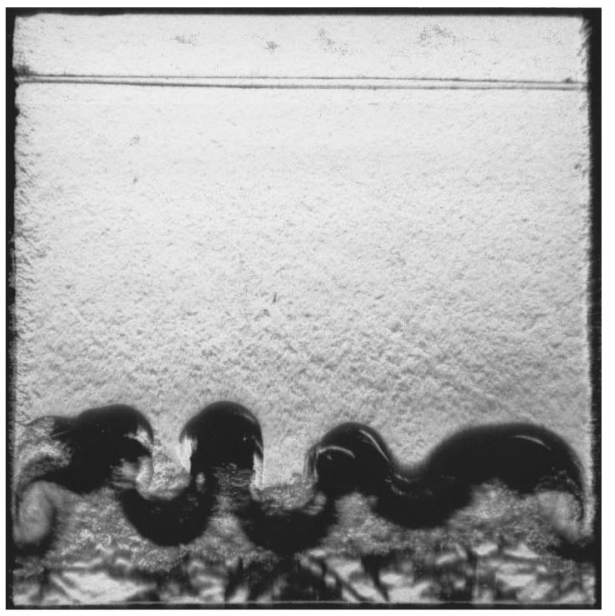

(a)

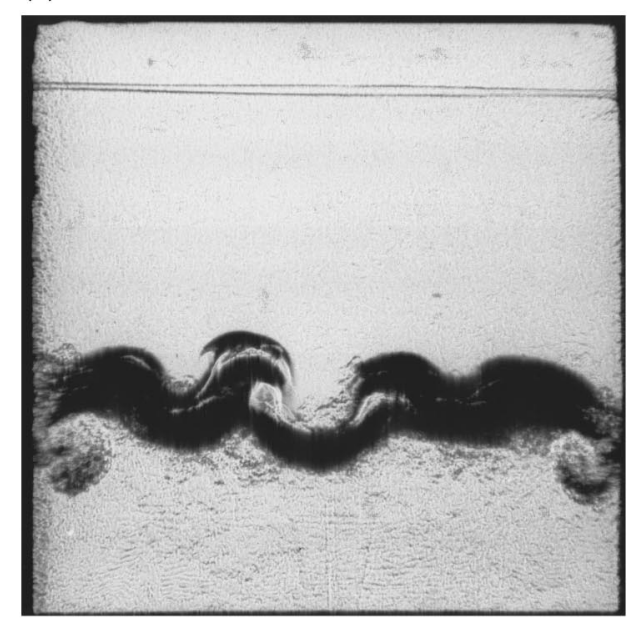

(b)

FIG. 21. (a): Air/SF 6 : $\tau=1.2 \mathrm{~s}, M_{i}=1.32$, and $t=2.11 \mathrm{~ms}$. (b) Air/Xe: $\tau=1.2 \mathrm{~s}, M_{i}=1.32$, and $t=3.70 \mathrm{~ms}$. Schlieren photographs.

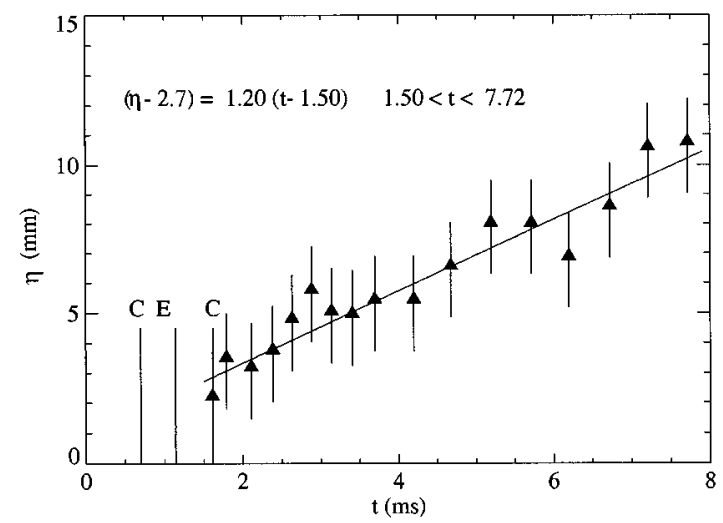

FIG. 22. Air/Xe: $\tau=1.2 \mathrm{~s}$, and $M_{i}=1.32$. Total amplitude versus time. Measurements from schlieren photographs: $\boldsymbol{\Delta}$ : measurements; —: linear leastsquares fit.

tures, has started to distort into a mushroom-like shape. The bottom of the photograph of the air/ $\mathrm{SF}_{6}$ interface shows a reverberating wave field between the interface and the end wall of the shock tube. Above it, the gray fine-scaled region lying roughly between the cross sections of the two wall vortices is believed to be the image of a vortex on the front window of the test section.

From the experiments it is observed that an air $/ \mathrm{SF}_{6}$ interface imaged at time $t$ is most similar to an air/xenon interface imaged at a time about $1.6 t$. At times $t \geqslant 3.15 \mathrm{~ms}$ for air/ $/ \mathrm{SF}_{6}$ and $t \geqslant 5.19 \mathrm{~ms}$ for air/xenon, the photographs suggest the presence of three-dimensional effects: In particular, some of the crests actually look like two spikes, one in front of the other. It is possible that these three-dimensional effects are confined to regions near the windows and do not occur in the mean flow.

In conclusion, the schlieren images show that air $/ \mathrm{SF}_{6}$ and air/xenon interfaces look very similar at comparable times. Therefore, the qualitative differences between the radiographs of air/xenon interfaces and the schlieren images of air/ $\mathrm{SF}_{6}$ interfaces are due to the difference between the physical processes upon which the two imaging techniques are based (schlieren is sensitive to the density gradients in the field of view, whereas the signal recorded by x-ray imaging is a direct function of the density of the test gas), and not to a different response to the impulsive acceleration between the two interfaces.

The results from the schlieren experiments are summarized in Figs. 22 and 23 and in Table VII. For the time interval between the interaction with the first expansion and the second reshock (second and third vertical lines in Fig. 23), Brouillette ${ }^{13}$ found growth rates for the "total" amplitude (i.e., without modal decomposition) of the perturbations on an air/ $\mathrm{SF}_{6}$ interface accelerated by a $M_{i}=1.32$ shock wave of $5.45 \mathrm{~m} / \mathrm{s}$ [the value predicted by Eq. (3) being 3.01 $\mathrm{m} / \mathrm{s}]$. Brouillette' $\mathrm{s}^{30}$ data for times subsequent to the interaction with the second reshock are also shown in Fig. 23 (Brouillette limited the analysis to the "early" times because of the grossly nonlinear behavior exhibited after the interaction with the second reshock). From the schlieren pictures of the present investigation, for $M_{i}=1.32$ the growth rate of the "total" amplitude on an air $/ \mathrm{SF}_{6}$ interface after the interac- 


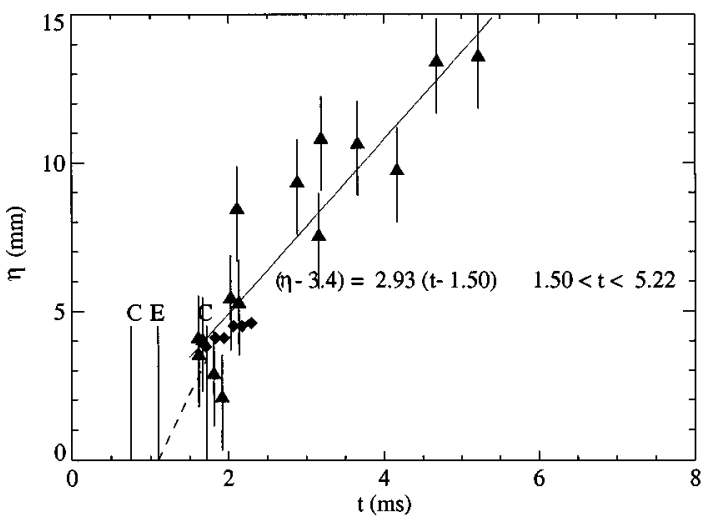

FIG. 23. Air/SF $: \tau=1.2 \mathrm{~s}$, and $M_{i}=1.32$. Total amplitude versus time. Measurements from schlieren photographs: $\boldsymbol{\Lambda},-$ : present measurements and least-squares fit; $\bullet,---$ : Brouillette. ${ }^{13,30}$

tion with the second reshock is measured to be $2.93 \mathrm{~m} / \mathrm{s}$. Brouillette's ${ }^{30}$ late time data compare favorably, within experimental error, to the portion of the present data taken at corresponding times. Measurements from schlieren pictures of an air/xenon interface accelerated by an $M_{i}=1.32$ shock, taken after the interaction with the second reshock, indicate a "total" amplitude growth rate of $1.20 \mathrm{~m} / \mathrm{s}$ (Fig. 22). The "total" amplitude can also be measured from the radiographs of the air/xenon interface by reconstructing a filtered mean interface shape using only the first six modes (Fig. 24). In this case, the perturbation growth rate after interaction with the second reshock is found to be $1.32 \mathrm{~m} / \mathrm{s}$.

The agreement between the "total" amplitude growth rates for an air/xenon interface measured by the schlieren $(1.2 \mathrm{~m} / \mathrm{s})$ and the x-ray $(1.32 \mathrm{~m} / \mathrm{s})$ techniques is acceptable. The comparison between present measurements of "total" amplitude for an air/ $\mathrm{SF}_{6}$ interface and the previous ones ${ }^{30}$ (both by the schlieren method) is also acceptable. In particular it must be noted that, even though in the present work each datum corresponds to a different run (with comparatively large scatter from experiment to experiment), while in Brouillette's work ${ }^{13,30}$ the data were all acquired in one run by means of high speed cinematography, the values of the amplitudes measured at the same times in the present work and in Brouillette's ${ }^{13,30}$ are actually very close (of the order of $4.5 \mathrm{~mm}$ ).

\section{B. Multi-scale interfaces}

Nominally flat interfaces are generated by letting $8.0 \mathrm{~s}$ elapse between the completion of plate retraction and shock arrival at the interface. For these interfaces the growth rate of short wavelength disturbances is large, as seen in Eq. (2) (for

TABLE VII. Total amplitude growth rates for single-scale interfaces.

\begin{tabular}{cccc}
\hline \hline & \multicolumn{2}{c}{ Air/Xe } & \\
\cline { 2 - 3 } & $\mathrm{X}$ ray & Schlieren & Air/SF \\
Schlieren \\
$M_{i}$ & $\frac{d \eta}{d t}(\mathrm{~m} / \mathrm{s})$ & $\frac{d \eta}{d t}(\mathrm{~m} / \mathrm{s})$ & $\frac{d \eta}{d t}(\mathrm{~m} / \mathrm{s})$ \\
\hline 1.32 & 1.32 & 1.20 & 2.93 \\
\hline \hline
\end{tabular}

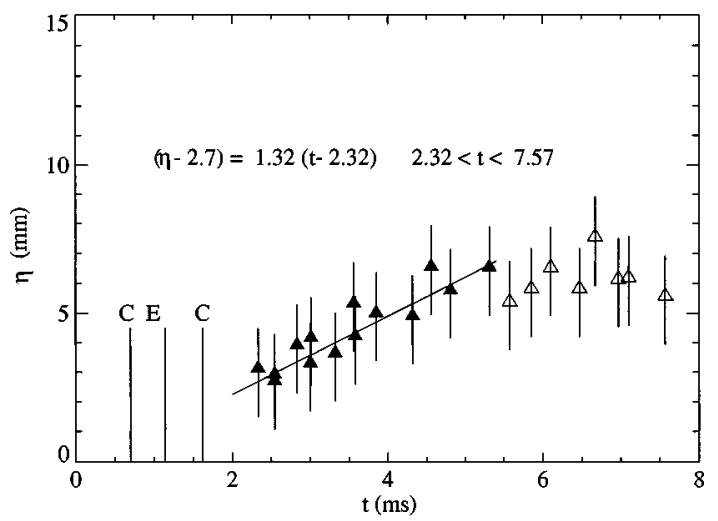

FIG. 24. Air/Xe: $\tau=1.2 \mathrm{~s}$, and $M_{i}=1.32$. Total amplitude versus time. Measurements from radiographs: $\boldsymbol{\Lambda}$ : data not affected by interface flattening; $\triangle$ : data adversely affected by interface flattening; —: linear least-squares fit.

a disturbance of wave number $k=10 \mathrm{~cm}^{-1}$ and initial amplitude $\eta=0.1 \mathrm{~mm}$ on a discontinuous air/Xe interface accelerated by a $M_{i}=1.32$ shock wave, the impulsive model predicts a growth rate of $6.2 \mathrm{~m} / \mathrm{s})$. On the other hand, the thickness-to-wavelength ratio is large, yielding a large value of the growth reduction factor, $\psi$, for the interface (of the order of 20 for a perturbation of the same wave number on an interface of thickness $\delta=1 \mathrm{~cm}$ ). The net result is that the interface thickens rather slowly. It looks essentially flat throughout the process (except for the distortions induced on its edges by the wall effects), and its thickness increases with time, in a manner similar to that for the single-scale interfaces.

\section{X-ray imaging and densitometry}

The interface remains essentially unchanged until some time after interaction with the reflected shock, when wall effects become large, and affect the whole span of the interface.

Figure 25 is the image of a multi-scale interface, taken at $t=6.08 \mathrm{~ms}$. Wall vortices have appeared by this time, inducing some deformation on less than one-third of the span of the interface. The interface thickness has grown to a value of about $14 \mathrm{~mm}$. By $t=11.07 \mathrm{~ms}$ (Fig. 26) the distortions at the

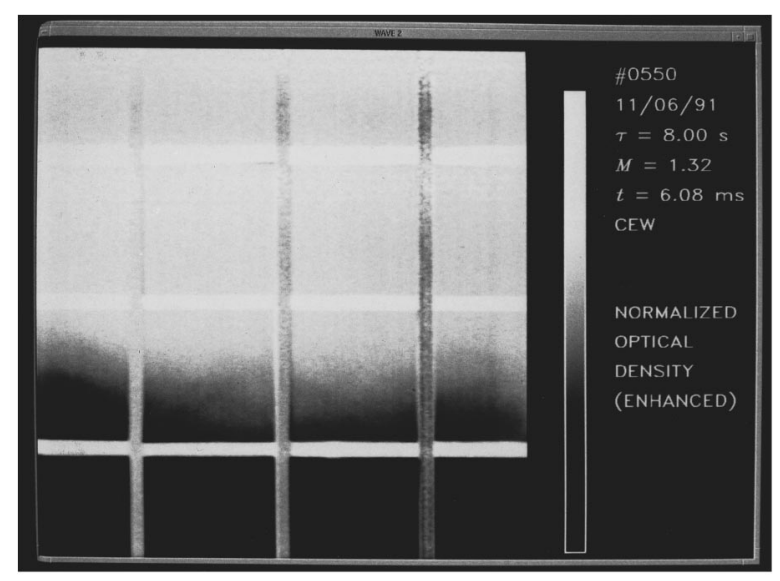

FIG. 25. Air/Xe. Run \#0550: $\tau=8.0 \mathrm{~s}, M_{i}=1.32$, and $t=6.08 \mathrm{~ms}$. Optical density of the radiograph. 


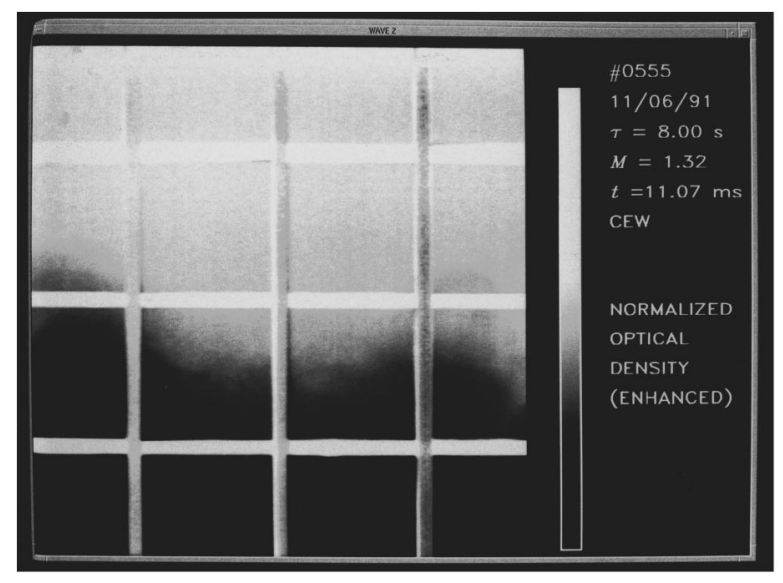

FIG. 26. Air/Xe. Run \#0555: $\tau=8.0 \mathrm{~s}, M_{i}=1.32, t=11.07 \mathrm{~ms}$. Optical density of the radiograph.

sides, induced by the images of the wall vortices, have become larger, but the middle of the interface is still essentially flat.

The interface thickness as a function of time is given in Fig. 27 for $M_{i}=1.32$, and Fig. 28 for $M_{i}=1.52$. The growth rates are $1.03 \mathrm{~m} / \mathrm{s}$ and $1.68 \mathrm{~m} / \mathrm{s}$, respectively. For $M_{i}=1.32$ the growth is maintained all the way to $10.5 \mathrm{~ms}$, whereas for $M_{i}=1.52$ saturation is observed after about $7 \mathrm{~ms}$. In both cases the thickness growth rate is smaller than the rate of increase of the thickness of the single-scale interfaces. This may be due to both the presence of large wavelength perturbations in the single-scale case, and larger values of the initial thickness (with correspondingly larger values of the growth reduction factor, $\psi$, at all wavelengths) in the multiscale case.

\section{Schlieren visualization}

Figure 29 shows schlieren photographs of an air/ $\mathrm{SF}_{6}$ interface and an air/xenon interface imaged at $t=3.14 \mathrm{~ms}$ and $t=6.20 \mathrm{~ms}$, respectively. In both cases the wall vortices and the "humps" they induce on the sides of the interface are clearly evident. In the air/ $\mathrm{SF}_{6}$ picture, the gray region below

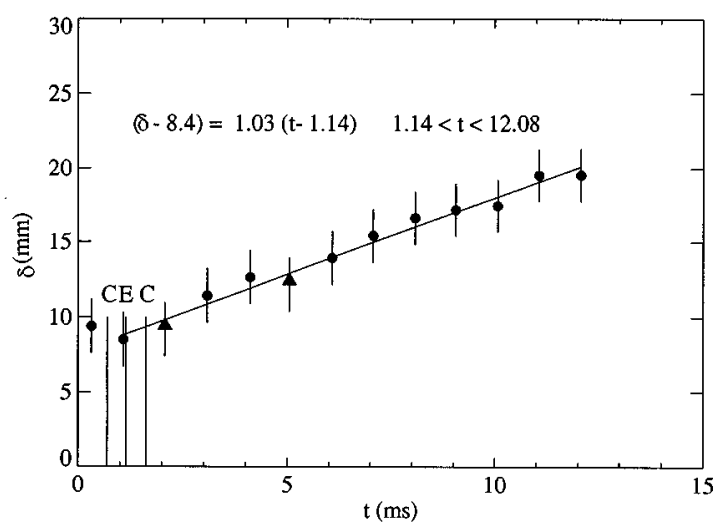

FIG. 27. Air/Xe: $M_{i}=1.32$, and $\tau=8.0$ s. Interface thickness versus time. Measurements from radiographs: $\boldsymbol{\Delta}$ : uniform Xe-density asymptote; non-uniform Xe-density asymptote; — : linear least-squares fit.

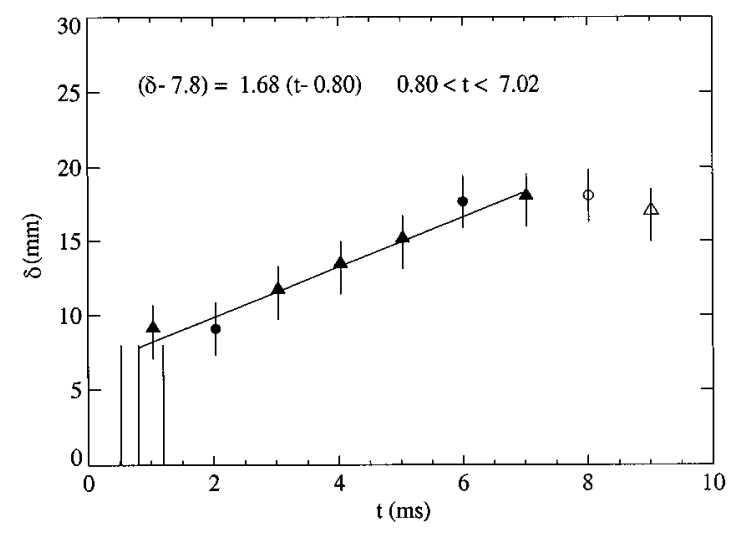

FIG. 28. Air/Xe: $M_{i}=1.52$ and $\tau=8.0 \mathrm{~s}$. Interface thickness versus time. Measurements from radiographs: $\boldsymbol{\Lambda}, \triangle$ : uniform Xe-density asymptote; $\bigcirc, \bigcirc$ : non-uniform Xe-density asymptote; $\boldsymbol{\Lambda}, \boldsymbol{O}$ : data not affected by interface flattening; $\triangle, \bigcirc$ : data adversely affected by interface flattening; linear least-squares fit.

the interface represents the wall vortex on the front window of the test section. A similar region, although less clear, is visible in the air/xenon photograph.

As time increases, the wall vortices and the perturbations they induce grow larger; the central portion of the interface

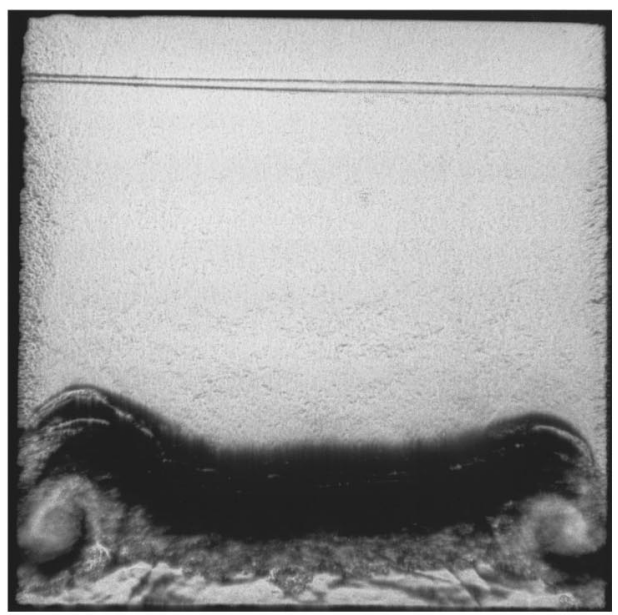

(a)

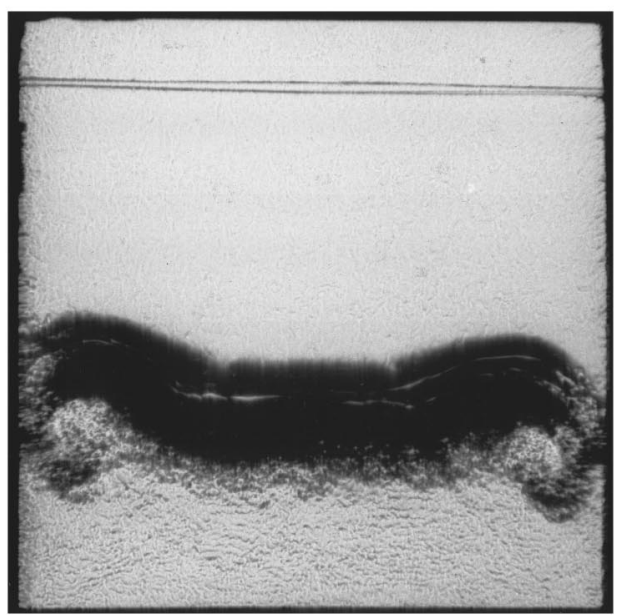

(b)

FIG. 29. (a): Air/SF $26: \tau=8.0 \mathrm{~s}, M_{i}=1.32$, and $t=3.14 \mathrm{~ms}$. (b) Air/Xe: $\tau=8.0 \mathrm{~s}, M_{i}=1.32$, and $t=6.20 \mathrm{~ms}$. Schlieren photographs. 


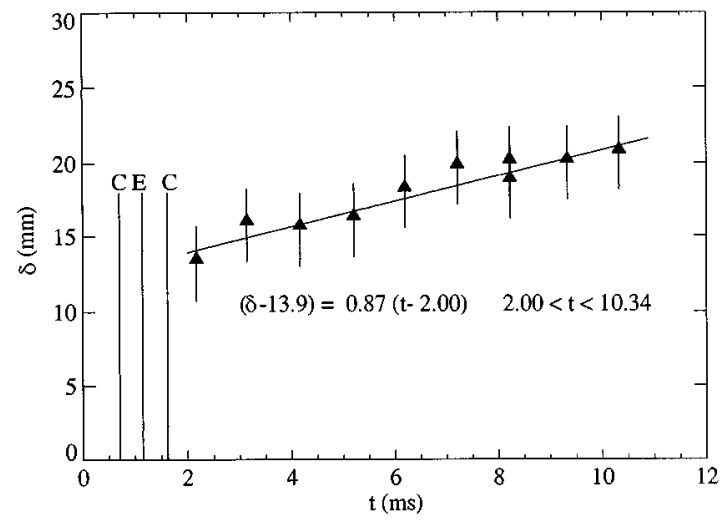

FIG. 30. Air/Xe: $\tau=8.0 \mathrm{~s}$, and $M_{i}=1.32$. Thickness versus time. Measurements from schlieren photographs: $\mathbf{\Delta}$ : measurements; - : linear leastsquares fit.

stays essentially flat. The wall vortices induce larger "humps" on the air/ $\mathrm{SF}_{6}$ interface than they do on the air/ xenon. To explain the difference in magnitude of the wall effects between xenon and $\mathrm{SF}_{6}$, it is best to first look at the interaction of a shock wave reflected from the end wall of a shock tube with the boundary layer generated by the incident wave,$^{32}$ in the absence of any interface. Depending on the incident Mach number and the ratio of specific heats $(\gamma)$ of the gas, one of the following situations may arise: The fluid within the boundary layer flows along the wall, simply crossing the reflected shock; the fluid within the boundary layer collects in a "bubble" immediately adjacent to the wall and an oblique shock forms between the bubble and the foot of the reflected shock; a bubble forms, and two oblique shocks form (bifurcation), between the bubble and the foot of the reflected shock. Bifurcation is more severe for low- $\gamma$ gases than for high. If the reshock is refracted by a gas interface, shock bifurcation may occur in the heavy gas. ${ }^{33}$ In the present experiments, shock bifurcation is never observed (by $\mathrm{x}$-ray or schlieren imaging) in either xenon or $\mathrm{SF}_{6}$. When the reflected shock interacts with the boundary layer within the interface, the velocity distribution inside the boundary layer represents a distortion of the interface, whose amplitude starts to grow upon shock-acceleration. If the reshock interacts with a heavy-light interface, it first causes a phase reversal of the distortion, which takes on the shape of spike of heavy fluid penetrating into the light one. At subsequent times, the spike rolls up into a vortex, as those observed in the present experiments. As for bifurcation, wall vortex effects are stronger for low- $\gamma$ gases than for high. ${ }^{34}$

As the wall effects grow larger, the distinction between the otherwise undisturbed interface and the wall vortex on the windows becomes more difficult. Throughout the process, an air $/ \mathrm{SF}_{6}$ interface at time $t$ best resembles an air/ xenon interface at time about $2 t$, as opposed to a time of about $1.6 t$ observed for the single-scale interfaces. The results of thickness measurements from schlieren images for a multi-scale air/xenon interface accelerated by a $M_{i}=1.32$ shock are presented in Fig. 30; results for an air/SF ${ }_{6}$ interface are shown in Fig. 31. The thickness growth rates are measured to be $0.87 \mathrm{~m} / \mathrm{s}$ for the air/xenon and $0.83 \mathrm{~m} / \mathrm{s}$ for the air/SF 6 interfaces, respectively.

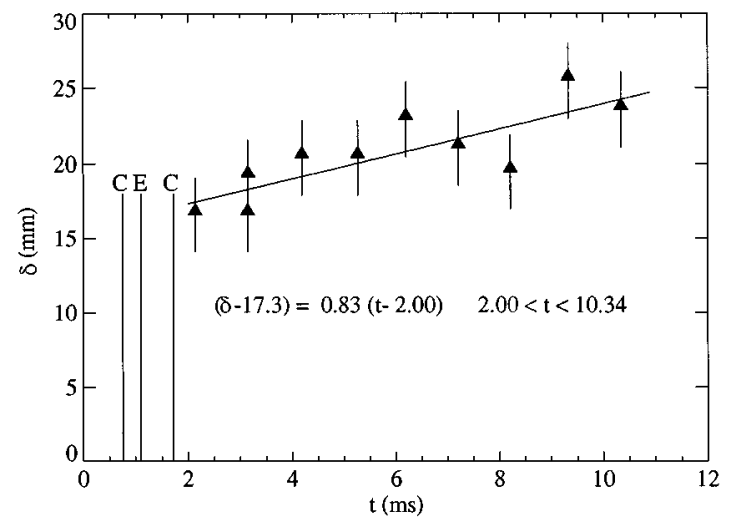

FIG. 31. Air/SF $\mathrm{SF}_{6}: \tau=8.0 \mathrm{~s}$, and $M_{i}=1.32$. Thickness versus time. Measurements from schlieren photographs: $\mathbf{\Delta}$ : measurements; - - linear leastsquares fit.

In his investigation of a multi-scale air/ $/ \mathrm{SF}_{6}$ interface (prepared with an 8-10 s delay between plate retraction and shock arrival) Brouillette ${ }^{12}$ found a postshock thickness of 31 $\mathrm{mm}$ and growth rates of 1.3 and $1.0 \mathrm{~m} / \mathrm{s}$, for $M_{i}=1.32$. Thus, as with the amplitude growth rates on single-scale interfaces, there is fairly good agreement between the thickness growth rate measured from the radiographs $(1.03 \mathrm{~m} / \mathrm{s})$ and from the schlieren photographs $(0.87 \mathrm{~m} / \mathrm{s})$ for an air/xenon interface. The agreement between the present $(0.83 \mathrm{~m} / \mathrm{s})$ and Brouillette's $^{12}(1.3,1.0 \mathrm{~m} / \mathrm{s})$ schlieren measurements of thickness growth rates for an air/SF 6 interface is also acceptable. The discrepancy between the present schlieren measurement of the postshock interface thickness $(\approx 10 \mathrm{~mm})$ and Brouillette's ${ }^{12}(\approx 30 \mathrm{~mm})$ indicates a different adjustment of the schlieren system sensitivity. Further evidence of this difference comes from the presence of a bright caustic line in the present schlieren images, not visible in Brouillette's. ${ }^{12,14}$

A plot of the correlation proposed by Brouillette ${ }^{12}$ [Eq. (8)] is presented in Fig. 32, which shows that the scatter in the present measurements is significantly smaller than in the

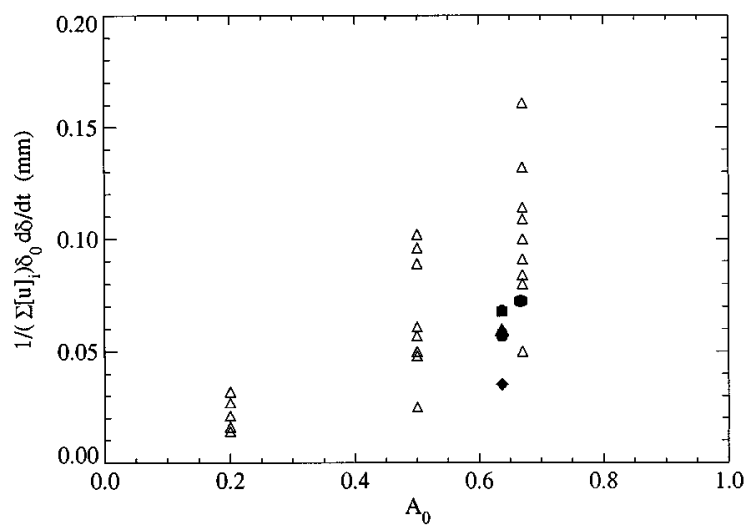

FIG. 32. Thickness growth rate correlation [Eq. (8)]: $\operatorname{air} / \mathrm{Xe}, \tau=1.2 \mathrm{~s}$, $M_{i}=1.32, \mathrm{x}$ ray; $\bullet$ air $/ \mathrm{Xe}, \tau=1.2 \mathrm{~s}, M_{i}=1.52$, x ray; $\Delta$ : air $/ \mathrm{Xe}, \tau=8.0 \mathrm{~s}$, $M_{i}=1.32$, x ray; $: \operatorname{air} / \mathrm{Xe}, \tau=8.0 \mathrm{~s}, M_{i}=1.52$, x ray; $\mathbf{a i r} / \mathrm{Xe}, \tau=8.0 \mathrm{~s}$, $M_{i}=1.32$, schlieren; $:$ air $/ \mathrm{SF}_{6}, \tau=8.0 \mathrm{~s}, M_{i}=1.32$, schlieren; $\triangle$ : Brouillette. $^{12}$ 
TABLE VIII. Thickness growth rates and correlation factor $\chi$ [Eq. (8)].

\begin{tabular}{cccccccc}
\hline \hline & & \multicolumn{3}{c}{ Air/Xe } & \multicolumn{2}{c}{ Air/SF } \\
\cline { 3 - 8 } & & \multicolumn{2}{c}{ X ray } & \multicolumn{2}{c}{ Schlieren } & \multicolumn{2}{c}{ Schlieren } \\
$\tau(\mathrm{s})$ & $M_{i}$ & $\frac{d \delta}{d t}(\mathrm{~m} / \mathrm{s})$ & $\chi$ & $\frac{d \delta}{d t}(\mathrm{~m} / \mathrm{s})$ & $\chi$ & $\frac{d \delta}{d t}(\mathrm{~m} / \mathrm{s})$ & $\chi$ \\
\hline 1.2 & 1.32 & 1.68 & 0.068 & & & & \\
1.2 & 1.52 & 1.86 & 0.035 & & & 0.072 \\
8.0 & 1.32 & 1.03 & 0.057 & 0.87 & 0.068 & 0.83 & 0.072 \\
8.0 & 1.52 & 1.68 & 0.057 & & & & \\
\hline \hline
\end{tabular}

previous ones. ${ }^{12}$ From the present data (including both single-scale and multi-scale interfaces, with $N=3$ ), an average value of $\chi=0.060 \pm 0.01 \mathrm{~mm}$ was found. A slightly different model,

$$
\frac{d \delta}{d t}=\chi \sum_{i=0}^{N-1} \frac{A_{i}}{\delta_{i}^{\prime}}\left|[v]_{i}\right|,
$$

has been tried to take into account the variations of the Atwood number and of the thickness due to each wave, but the factor $\chi$ turns out to have the same value, and only slightly less scatter.

As for the single-scale interfaces, no power law behavior, after the linear one, can be deduced from the existing data. Table VIII presents a summary of the thickness growth rates and correlation factors from the present experiments.

\section{CONCLUSIONS}

X-ray imaging is successfully applied to the visualization of gas flow in a shock tube. A new densitometry technique is demonstrated, and applied to the study of shockaccelerated, unstable gas interfaces. All the interfaces observed in the present work are initially continuous, with finite density gradients across them. Two types of interface, single scale and multi-scale, are studied. The gases at the interface are always air and xenon, this latter chosen for its unique x-ray absorption properties. Shock waves of two different Mach numbers are used in a geometry allowing multiple shock-interface interactions.

Growth rates of the spatial modes of the mean interface shapes are generally smaller than predicted by the impulsive model in its modified version for multiple wave-interface interactions and finite density gradients. This is expected since the perturbations are observed when the small amplitude approximation is no longer verified.

The thickness growth after the reshock is due mostly to the random acoustic disturbances generated by the interaction of the reshock with the boundary layer. The measurements yield growth rates comparable to those previously reported. The x-ray densitometry technique produces, as expected, smaller thickness values than schlieren imaging had in the past. The thickness growth rate of single scale interfaces is larger than that of the multi-scale interfaces.

The agreement between the present x-ray and schlieren visualizations and that between the present schlieren experiments and Brouillette's validate the x-ray imaging technique. In particular, satisfactory agreement was obtained between the growth rates of the "total" amplitude of air/xenon inter- faces measured by the schlieren and x-ray techniques; the agreement between the growth rates of the thickness of air/ xenon interfaces measured by the schlieren and x-ray techniques was also acceptable. Finally, the agreement between schlieren measurements of the thickness growth rates of air/ $\mathrm{SF}_{6}$ interfaces from the present study and Brouillette's ${ }^{12}$ was satisfactory.

\section{ACKNOWLEDGMENT}

This research was supported by the U.S. Department of Energy, Lawrence Livermore National Laboratory, under project agreement DOE W-7405-ENG-48.

${ }^{1}$ Lord Rayleigh, "Investigation of the character of the equilibrium of an incompressible heavy fluid of variable density," in Scientific Papers (Dover, New York, 1900), Vol. 2.

${ }^{2}$ G. I. Taylor, "The instability of liquid surfaces when accelerated in a direction perpendicular to their planes, I," Proc. R. Soc. London, Ser. A 201, 192 (1950).

${ }^{3}$ R. D. Richtmyer, "Taylor instability in shock acceleration of compressible fluids," Comm. Pure Appl. Math. 8, 297 (1960).

${ }^{4} \mathrm{E}$. Meshkov, "Instability of the interface of two gases accelerated by a shock-wave," Sov. Fluid Dynam. 4, 101 (1969).

${ }^{5}$ L. D. Landau and E. M. Lifshitz, Fluid Mechanics (Pergamon, New York, 1959), p. 477.

${ }^{6}$ S. Chandrasekhar, Hydrodynamic and Hydromagnetic Stability (Oxford University Press, Oxford, 1961), p. 481

${ }^{7}$ A. M. Abd-El-Fatah and L. F. Henderson, "Shock waves at a fast-slow gas interface," J. Fluid Mech. 86, 15 (1978).

${ }^{8}$ A. M. Abd-El-Fatah and L. F. Henderson, "Shock waves at a slow-fast gas interface,'” J. Fluid Mech. 89, 79 (1978).

${ }^{9}$ C. J. Catherasoo and B. Sturtevant, "Shock dynamics in non-uniform media," J. Fluid Mech. 127, 539 (1983).

${ }^{10}$ D. W. Schwendeman, "Numerical shock propagation in non-uniform media," J. Fluid Mech. 188, 383 (1988).

${ }^{11}$ Y. Tang, Q. Zhang, and D. H. Sharp, "Small amplitude theory of Richtmyer-Meshkov instability," Phys. Fluids 6, 1856 (1994).

${ }^{12}$ M. Brouillette and B. Sturtevant, "Growth induced by multiple shock waves normally incident on plane gaseous interfaces," Phys. D 37, 248 (1989).

${ }^{13} \mathrm{M}$. Brouillette and B. Sturtevant, "Experiments on the RichtmyerMeshkov instability; single-scale perturbations on a continuous interface," J. Fluid Mech. 263, 271 (1994).

${ }^{14}$ M. Brouillette and B. Sturtevant, "Experiments on the RichtmyerMeshkov instability: Small scale perturbations on a plane interface," Phys. Fluids A 5, 916 (1993).

${ }^{15}$ S. G. Zaitsev, E. V. Lazareva, V. V. Chernukha, and V. M. Belyaev, "Intensification of mixing at the interface between media of different densities upon the passage of a shock wave through it," Sov. Phys. Dokl. 30, 579 (1985).

${ }^{16} \mathrm{~T}$. Pham, "Numerical studies of incompressible Richtmyer-Meshkov instability in a stratified fluid," Ph.D. thesis, California Institute of Technology, 1990.

${ }^{17}$ G. Fraley, "Rayleigh-Taylor instability for a normal shock wave-density discontinuity interaction," Phys. Fluids 29, 376 (1986).

${ }^{18} \mathrm{~K}$. Mikaelian, "Growth rate of the Richtmyer-Meshkov instability at shocked interfaces,'” Phys. Rev. Lett. 71, 2903 (1993).

${ }^{19}$ R. L. Holmes, J. W. Grove, and D. H. Sharp, "Numerical investigation of Richtmyer-Meshkov instability using front tracking," J. Fluid Mech. 301, 51 (1995).

${ }^{20} \mathrm{R}$. Benjamin, "Experimental observations of shock stability and shockinduced turbulence," in Advances in compressible turbulent mixing, edited by W. P. Dannevick, A. C. Buckingham, and C. E. Leith (National Technical Information Service, Springfield, VA, 1992), p. 341.

${ }^{21}$ R. Benjamin, D. Besnard, and J. F. Haas, "Shock and reshock of an unstable interface,'” Report LA-UR 92-1185, Los Alamos National Laboratory (1993).

${ }^{22}$ K. O. Mikaelian, "Richtmyer-Meshkov instabilities in stratified fluids," Phys. Rev. A 31, 410 (1985).

${ }^{23}$ R. E. Duff, F. H. Harlow, and C. W. Hirt, "Effects of diffusion on inter- 
face instability between gases," Phys. Fluids 5, 417 (1962).

${ }^{24}$ K. O. Mikaelian, "Simulation of the Richtmyer-Meshkov instability and turbulent mixing in shock-tube experiments," unpublished report, UCID21328, Lawrence Livermore National Laboratory (1988).

${ }^{25}$ V. A. Andronov, S. M. Bakhrakh, E. E. Meshkov, V. N. Mokhov, V. V. Nikiforov, A. V. Pevnitskii, and A. I. Tolshmyakov, "Turbulent Mixing at Contact Surface Accelerated by Shock Waves,' Sov. Phys. JETP 44, 424 (1976).

${ }^{26}$ V. A. Andronov, S. M. Bakhrakh, E. E. Meshkov, V. V. Nikiforov, A. V. Pevnitskii, and A. I. Tolshmyakov, "An experimental investigation and numerical modeling of turbulent mixing in one-dimensional flows," Sov. Phys. Dokl. 27, 393 (1982).

${ }^{27}$ G. I. Barenblatt, "Self-similar turbulence propagation from an instantaneous plane source," in Non-Linear Dynamics and Turbulence, edited by G. I. Barenblatt, G. Ioos, and D. D. Joseph (Pitman, Boston, 1983), p. 48.
${ }^{28}$ K. O. Mikaelian, "Turbulent mixing generated by Rayleigh-Taylor and Richtmyer-Meshkov instabilities," Physica D 36, 343 (1989).

${ }^{29}$ K. I. Read and D. L. Youngs, "Experimental investigation of turbulent mixing by Rayleigh-Taylor instability," AWRE Report 0-11/83 (1983).

${ }^{30} \mathrm{M}$. Brouillette, "On the interaction of shock waves with contact surfaces between gases of different densities,' Ph.D. thesis, California Institute of Technology, 1989.

${ }^{31}$ W. H. McMaster, "Compilation of x-ray cross sections," Report UCRL50174, Lawrence Livermore National Laboratory (1969).

${ }^{32} \mathrm{H}$. Mark, "The interaction of a reflected shock wave with the boundary layer in a shock tube,' J. Aero. Sci. 24, 304 (1957).

${ }^{33}$ R. V. Hess, "Interaction of moving shocks and hot layers," NACA TN4002 (1957).

${ }^{34} \mathrm{M}$. Brouillette and R. Bonazza, "Experiments on the Richtmyer-Meshkov instability: Wall effects and wave phenomena," submitted to Phys. Fluids. 